\title{
Calbindin and Parvalbumin Cells in Monkey VPL Thalamic Nucleus: Distribution, Laminar Cortical Projections, and Relations to Spinothalamic Terminations
}

\author{
E. Rausell,a C. S. Bae, ${ }^{b}$ A. Viñuela, ${ }^{a}$ G. W. Huntley, ${ }^{c}$ and E. G. Jones \\ Department of Anatomy and Neurobiology, University of California at Irvine, Irvine, California 92717
}

The ventral posterior lateral nucleus (VPL) of the monkey thalamus was investigated by histochemical staining for cytochrome oxidase (CO) activity and by immunocytochemical staining for the calcium-binding proteins parvalbumin and $28 \mathrm{kDa}$ calbindin. Anterograde and retrograde tracing experiments were used to correlate patterns of differential distribution of $\mathrm{CO}$ activity and of parvalbumin and calbindin cells with the terminations of spinothalamic tract fibers and with the types of cells projecting differentially to superficial and deeper layers of primary somatosensory cortex (SI).

VPL is composed of CO-rich and CO-weak compartments. Cells are generally smaller in the CO-weak compartment. Parvalbumin-immunoreactive cells and parvalbumin-immunoreactive medial lemniscal fiber terminations are confined to the CO-rich compartment. Calbindin-immunoreactive cells are found in both the CO-rich and CO-weak compartments. The CO-weak compartment, containing only calbindin cells, forms isolated zones throughout VPL and expands as a cap covering the posterior surface of the ventral posterior medial nucleus (VPM). Spinothalamic tract terminations tend to be concentrated in the CO-weak compartment, especially in the posterior cap. Other CO-weak, parvalbumin-negative, calbindin-positive nuclei, including the posterior, ventral posterior inferior, and anterior pulvinar and the small-celled matrix of VPM are also associated with concentrations of spinothalamic and caudal trigeminothalamic terminations.

Parvalbumin cells are consistently larger than calbindin cells and are retrogradely labeled only after injections of tracers in middle and deep layers of SI. The smaller calbindin cells are the only cells retrogradely labeled after placement of retrograde tracers that primarily involve layer I of SI.

The compartmental organization of VPL is similar to but less rigid than that previously reported in VPM. VPL and VPM relay cells projecting to different layers of SI cortex can be distinguished by differential immunoreactivity for the two

\footnotetext{
Received Mar. 13, 1992; revised May 26, 1992; accepted May 28, 1992.

This work was supported by Grants NS 21377 and NS 22317 from the National Institutes of Health, United States Public Health Service. E.R. was the recipient of a Fullbright fellowship and a Fogarty international fellowship. G.W.H. was the recipient of a National Institute of Mental Health predoctoral fellowship. We thank Phong Nguyen for technical help.

Correspondence should be addressed to Dr. E. G. Jones at above address.

aPresent address: Department of Morphology, University Autonoma at Madrid, School of Medicine, 28209 Madrid, Spain.

'Present address: Department of Anatomy, Chonnam University Medical School, Seoul, Korea.

'Present Address: Department of Neurobiology, The Mount Sinai School of Medicine, New York, NY 10029-6574.

Copyright (C) 1992 Society for Neuroscience $0270-6474 / 92 / 124088-24 \$ 05.00 / 0$
}

calcium-binding proteins. The small-celled, CO-weak, calbindin-positive zones of VPL and VPM appear to form part of a wider system of smaller thalamic neurons unconstrained by traditional nuclear boundaries that are preferentially the targets of spinothalamic and caudal trigeminal inputs, and that may have preferential access to layer I of SI.

The mammalian thalamus has traditionally been viewed as made up of a series of nuclear subunits, each receiving the terminations of a particular afferent pathway and projecting to a functional area of the cerebral cortex (historically reviewed in LeGros Clark, 1932; Walker, 1938; Jones, 1985). In recent years, this timehonored principle has been extended and modified in a number of ways. New evidence shows that many, perhaps all, thalamic nuclei contain subpopulations of relay neurons that project differentially upon the cerebral cortex. Different neurons in a single nucleus may project to different areas, single neurons may project by branched axons to more than a single arca, and the axons of different cells may have terminations in different layers or different densities of terminations in the cortex (see reviews by Herkenham, 1986; Macchi, 1988). The identification of these different classes of thalamocortical relay neuron has proceeded along a number of lines. These include physiological identification in terms of receptive field and stimulus-response patterns (c.g., Gordon and Manson, 1967; Cleland et al., 1971; Chung et al., 1986a,b), structure-function correlations made by the intracellular labeling of physiologically identified neurons (e.g., Friedlander et al., 1981; Stanford et al., 1983; Yen et al., 1985), retrograde labeling by anatomical tracers applied to different areas and layers of the cerebral cortex (e.g., Macchi et al., 1984; Rausell and Avendaño, 1985; Minciacchi et al., 1986; Birnbacher and Albus, 1987; Morel and Imig, 1987; Avendaño et al., 1990; Hashikawa et al., 1991; Kaske et al., 1991), and immunocytochemistry for cell-specific proteins (e.g., Hendry et al., 1988; Hockfield and Sur, 1990). Immunocytochemical staining for the calcium-binding protein parvalbumin, and the $28 \mathrm{kDa}$, vitamin $\mathrm{D}$-dependent calcium-binding protein calbindin has been particularly fruitful in identifying separate populations of relay neurons, especially in the primate thalamus (Jones and Hendry, 1989).

In the somatosensory thalamus, composed of the ventral posterior and certain adjacent nuclei, retrograde tracing studies in carnivores (Penny et al., 1982; Rausell and Avendaño, 1985; Avendaño et al., 1990) revealed the presence of a population of cells of small diameter that project specifically to layer I of the somatosensory cortex. In a recent investigation in the monkey, a similar layer I-projecting, small-celled population was 
identified in the ventral posterior medial nucleus (VPM) in which the cutaneous and mucosal surfaces innervated by the trigeminal nerve are represented (Rausell and Jones, 1991a,b). Apart from their smaller size, these neurons could also be identified by immunoreactivity for calbindin and by being concentrated in a compartment of the nucleus characterized by weak histochemical staining for the mitochondrial enzyme cytochrome oxidase (CO). This small-celled, calbindin-rich and $\mathrm{CO}$-weak compartment of VPM forms a matrix of the nucleus in which are embedded elongated clusters of large- and medium-sized neurons projecting to deeper layers of the somatosensory cortex. These clusters or "rods" are further characterized by high CO activity and their contained relay neurons by immunoreactivity for parvalbumin and for the proteoglycan identified by the monoclonal antibody CAT301. As a clue to the functional significance of this compartmental organization of differentially projecting relay neurons, it was found that the matrix compartment is preferentially innervated by fibers ascending from the caudal nucleus of the spinal trigeminal complex (Rausell and Jones, 1991a) while the rod compartment is preferentially innervated by fibers arising in the principal sensory trigeminal nucleus (Jones et al., 1986a). These observations suggested the existence of parallel pathways for the relay of pain and temperature and for innocuous tactile information from the face to different layers of the somatosensory cortex.

Populations of calbindin- and parvalbumin-immunoreactive relay neurons have also been identified in the monkey ventral posterior lateral nucleus (VPL) (Jones and Hendry, 1989) in which the remainder of the body is represented (Mountcastle and Henneman, 1952; Mountcastle and Poggio, 1963; Jones and Friedman, 1982; Kaas et al., 1984). This subnucleus is CO rich, and in primates both spinothalamic and medial lemniscal fibers terminate in it (Mehler et al., 1960; Bowsher, 1961; Mehler, 1969; Kerr and Lippman, 1974; Kerr, 1975; Boivie, 1978, 1979; Applebaum et al., 1979; Berkley, 1980; Tracey et al., 1980; Jones et al., 1982; Asanuma et al., 1983; Burton and Craig, 1983; Mantyh, 1983; Apkarian and Hodge, 1989c). However, any relationship of the calbindin- and parvalbumin-immunoreactive cells to a compartmental organization or to the differential terminations of the two somatosensory pathways, and the possibility of their differential projections upon the somatosensory cortex have not been revealed. This article reports the results of such an investigation.

A preliminary account has appeared in abstract form (Rausell et al., 1991).

\section{Materials and Methods}

The experiments were carried out on 21 adult monkeys $(17 \mathrm{Macaca}$ fascicularis and 4 Macaca fuscata), wcighing $3.0-3.5 \mathrm{~kg}$. Ten of the animals were normal and had been used in previous immunocytochemical studies on VPM (Jones and Hendry, 1989; Rausell and Jones, 1991b). Of the other 11, 6 were used in combined immunocytochemical and tracing studies, involving injections of anterogradely or retrogradely transported Fast Blue (FB). The remaining five were used for anterograde or retrograde tracing studies involving injections of horseradish peroxidase (HRP).

Immunocytochemistry. Animals were deeply anesthetized with intravenous Nembutal and perfused through the left ventricle with $300 \mathrm{ml}$ saline, followed by 3 liters of a fixative containing $2.0 \%$ paraformaldehyde and 0.1 or $0.2 \%$ glutaraldehyde in $0.1 \mathrm{M}$ phosphate buffer $(\mathrm{pH}$ 7.4 ), and then successively with $10 \%, 15 \%$, and $20 \%$ sucrose in $0.1 \mathrm{M}$ phosphate buffer. The brains were removed, infiltrated with $30 \%$ sucrose, blocked, frozen in dry icc, and scctioned on a sliding microtome. The thalami were serially sectioned in the frontal, sagittal, or horizontal planes into six series of alternating $15 \mu \mathrm{m}$ and $30 \mu \mathrm{m}$ sections. Every

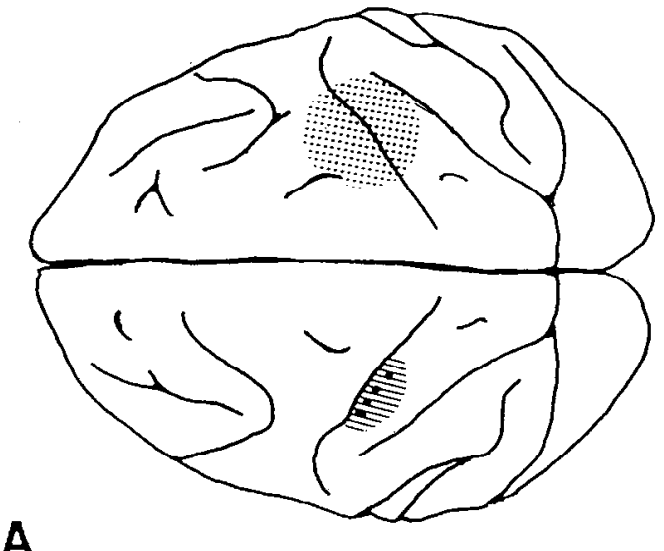

A

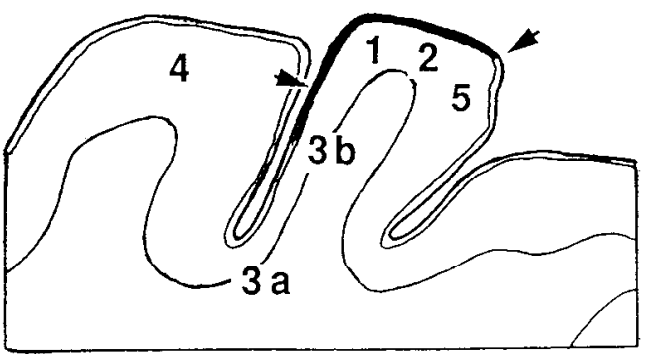

B

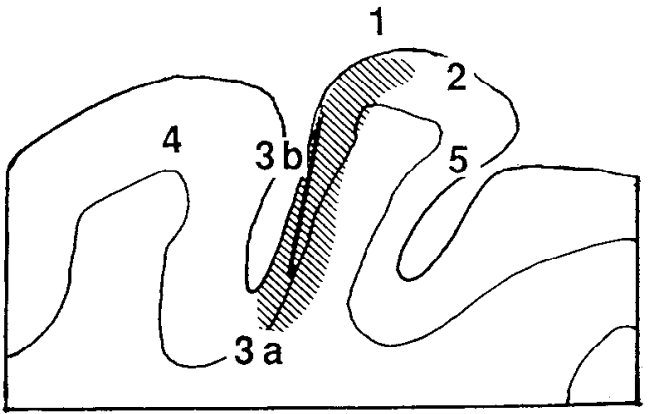

C

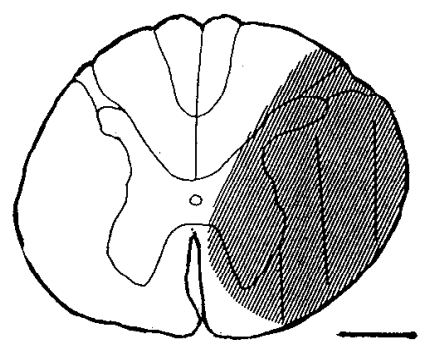

D

Figure 1 . Placements of retrogradely $(A-C)$ and anterogradely $(D)$ transported tracers in the cerebral cortex or spinal cord. A, Stippling over right pre- and postcentral gyri indicates maximal extent of spread of FB or HRP applied to surface of the cortex. Solid circles and hatching in left postcentral gyrus indicate injection sites and maximal spread of FB or HRP when injected via micropipettes into the physiologically localized hand representation. $B$ and $C$ show camera lucida drawings of sections taken through a brain in which FB was applied to the surface of the postcentral gyrus only (arrows, $B$ ) or injected into area $3 \mathrm{~b}(C) . D$ shows three micropipette tracks (lines) and maximal spread of HRP (hatching) when injected into the lateral and ventral funiculi at the second cervical segment of the spinal cord. Scale bars, $1 \mathrm{~mm}$. 
$15 \mu \mathrm{m}$ section was stained, free floating, for immunocytochemistry. Parvalbumin was localized by means of a sheep anti-parvalbumin antiserum (see Jones and Hendry, 1989) or a mouse monoclonal antibody (Sigma). Calbindin was localized with a rabbit anti-calbindin antiserum (see Jones and Hendry, 1989) that will also recognize $29 \mathrm{kDa}$ calretinin (Rogers, 1987). The monoclonal antibodies and antisera were diluted in $0.1 \mathrm{M}$ phosphate buffer ( $\mathrm{pH} 7.4$ ) containing $0.25 \%$ Triton $\mathrm{X}-100$ and $3 \%$ normal serum of the species in which the secondary antibody to be used was raised. Final dilutions of the primary antisera or antibodies were $1: 3000$. The sections were preincubated for $1-2 \mathrm{hr}$ in $0.1 \mathrm{M}$ phosphate buffer containing 3\% normal serum and $0.25 \%$ Triton X-100 and then transferred to the primary antibody-containing solution for 24-36 $\mathrm{hr}$ at $4^{\circ} \mathrm{C}$. The sections were then washed thoroughly in phosphate buffer and stained by the avidin-biotin-peroxidase complex method (ABC, Vectastain). Immunoreactivity was finally developed by incubation in a solution of $0.02 \% 3,3$ '-diaminobenzidine tetrahydrochloride and $0.001 \%$ hydrogen peroxide. The alternating series of $30 \mu \mathrm{m}$ sections was stained histochemically for cytochrome oxidase (CO) (Wong-Riley, 1979), or with $0.25 \%$ thionin. Controls for these and the subsequently described immunocytochemical procedures consisted of staining selected sections using preimmune serum in place of the primaries, and by staining using antibodies or antisera that had been subjected to preadsorption in an excess of parvalbumin (Sigma) or calbindin (Sigma). None of these procedures revealed other than a light, diffuse background, and no cells were stained.

In two of the normal animals, one Macaca fascicularis and one $\mathrm{Ma}$ caca fuscata, counts and measurements were made of the somal sizes of parvalbumin- and calbindin-immunoreactive neurons in VPL. Two series of 10 sections, each section separated by $180 \mu \mathrm{m}$ from its neighbors, were selected; one series was stained immunocytochemically for calbindin, and the other, for parvalbumin. In each section, areas measuring $0.70 \mathrm{~mm}^{2}$ or $0.77 \mathrm{~mm}^{2}$, as outlined by an eyepiece reticule, were selected from the center, medial, and anterodorsal aspects of VPL. All immunoreactive neuronal somata falling within the reticule were then counted, and the outlines of those containing and not containing a sectioned nucleus were separately traced with the camera lucida at $1000 \times$. Diameters and areas were then determined using a Zeiss, Zidas digitizing tablet. Differences between the sizes of parvalbumin- and calbindinimmunoreactive neurons were compared using Student's $t$ test and multiple analysis of variance (ANOVA). To calculate the number of cells per volume, the stereological formula of DeHoff and Rhines (1961; Montero and Zempel, 1986) was used.

Anterograde tracing. In two monkeys, Fast Blue (FB) was injected into the spinal cord (Fig. 1). The monkeys were anesthetized with intravenous Nembutal, and under aseptic conditions the neck muscles were detached from the occipital bone on one side to expose the posterior aspects of the second, third, and fourth cervical vertebrae. The laminae of one of these vertebrae were removed, and the dura mater was incised. A micropipette with a tip diameter of $20-30 \mu \mathrm{m}$, attached to a $10 \mu \mathrm{l}$ Hamilton syringe and coupled to an oil-filled hydraulic system, was introduced vertically into the anterolateral quadrant of the spinal cord at approximately the third cervical segment. A $5 \%$ aqueous solution of fast blue $(2-4 \mu \mathrm{l})$ was injected in one or more penetrations. After a 2 week survival period, the animals were deeply anesthetized and perfused

Figure 2. Trios of closely adjacent frontal sections through anterior $(A-C)$, middle $(D-F, G-I)$, and posterior $(J-L)$ levels of the thalamus of a normal monkey and stained for $\mathrm{CO}$ activity $(C O: A, D, G, J)$, parvalbumin immunoreactivity $(P A R V: B, E, H, K)$, or calbindin immunoreactivity $(C A L B: C, F, I, L)$. Arrows indicate the same blood vessels in each trio. Arrowheads in $A-C$ indicate lamina between centre médian nucleus $(C M)$ and VPM in which calbindin matrix of VPM first becomes evident. This expands as a CO-weak, parvalbumin-negative but calbindin-rich region $(s)$ at more posterior levels, becoming the posterior "cap" in $J-L$. Note CO-weak, parvalbumin-negative zones in VPL in $D-F$ and $G-I$ (open arrows in $G-I)$. Calbindin cells are scattered throughout VPL. For sections at a more posterior level, see Figure $4 G-$ $I$. In this and subsequent illustrations, approximate stereotaxic levels, derived from section thickness and position in a series, are shown at top left of each row. These provide an indication of anterior, middle, and posterior levels through VPL, which normally extends from approximately A6 anteriorly to A2 posteriorly. For other abbreviations, see Appendix. Scale bars, $1 \mathrm{~mm}(E$ also for $A, B$, and $D ; F$ also for $C$; $H$ also for $G, J$, and $K ; I$ also for $L$ ).
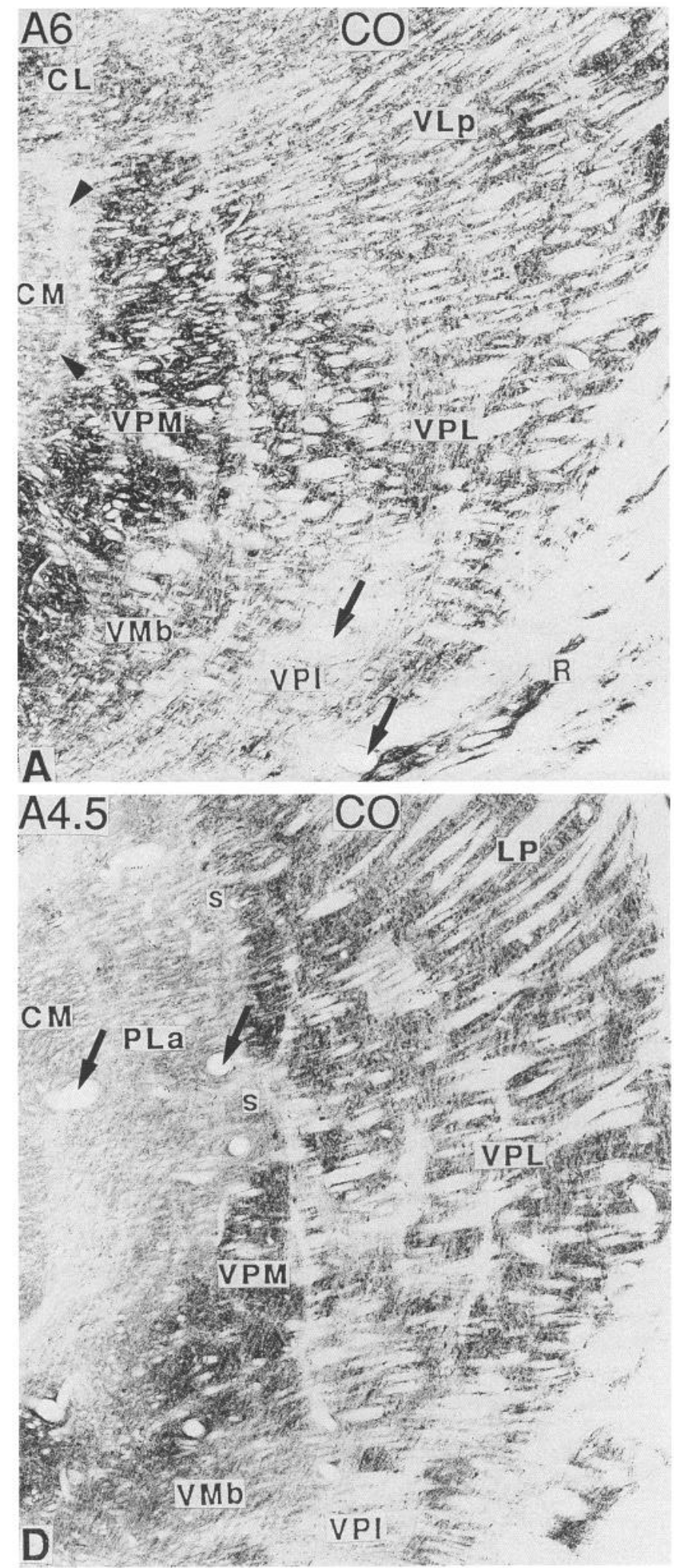

with a mixture of $4 \%$ paraformaldehyde and $0.2 \%$ glutaraldehyde in 0.1 $\mathrm{M}$ phosphate buffer. The brain and spinal cord were removed, blocked, and frozen in dry ice. Six series of alternating $15-\mu \mathrm{m}$ - and $30-\mu \mathrm{m}$-thick frontal or sagittal sections of the thalamus and transverse sections of the brainstem and spinal cord were collected in cold $0.1 \mathrm{M}$ phosphate buffer. The first $30 \mu \mathrm{m}$ section of each group of six sections was mounted quickly for immediate examination under the fluorescence microscope 

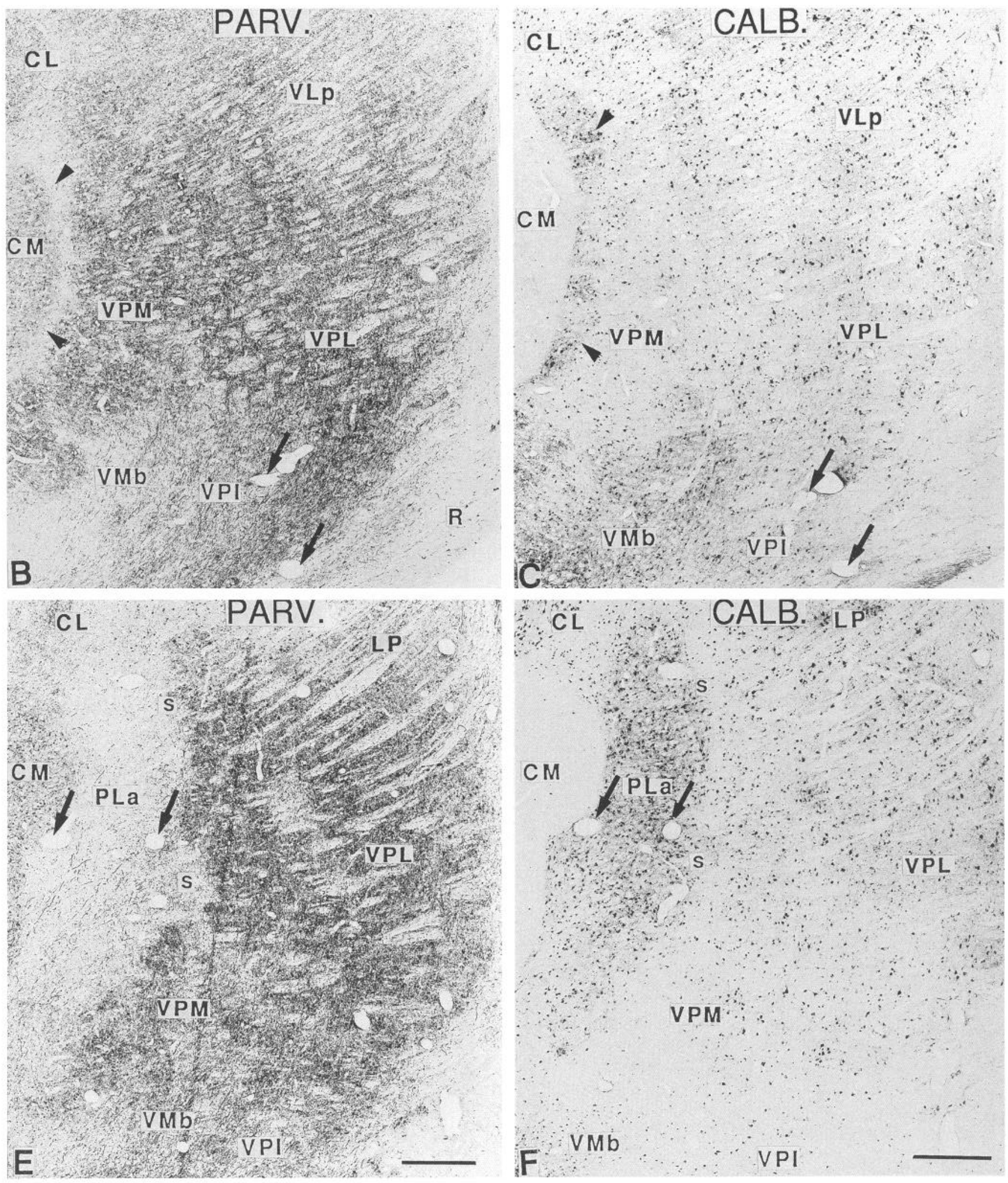

and photography of anterogradely labeled fibers. The adjacent $30 \mu \mathrm{m}$ section was stained with $0.25 \%$ thionin. The remaining $30 \mu \mathrm{m}$ sections were processed for $\mathrm{CO}$ histochemistry. The $15 \mu \mathrm{m}$ sections were stained immunocytochemically for the localization of parvalbumin or calbindin or for both of these simultaneously. For the localization of a single antigen, a procedure similar to that described above was followed, using the same dilutions of primary antibody but without the addition of

Triton X-100. Then, the sections were washed and incubated for $2 \mathrm{hr}$ at $4^{\circ} \mathrm{C}$ in a 1:200 solution of a fluorescein isothiocyanate (FITC)-conjugated donkey anti-rabbit or rhodamine isothiocyanate (RITC)-conjugated donkey anti-sheep secondary antibody (Chemicon). For colocalization of the two antigens, the sections were preincubated in $3 \%$ normal horse serum without Triton X-100 and then incubated in solutions containing 3\% normal horse serum and anti-calbindin and anti- 

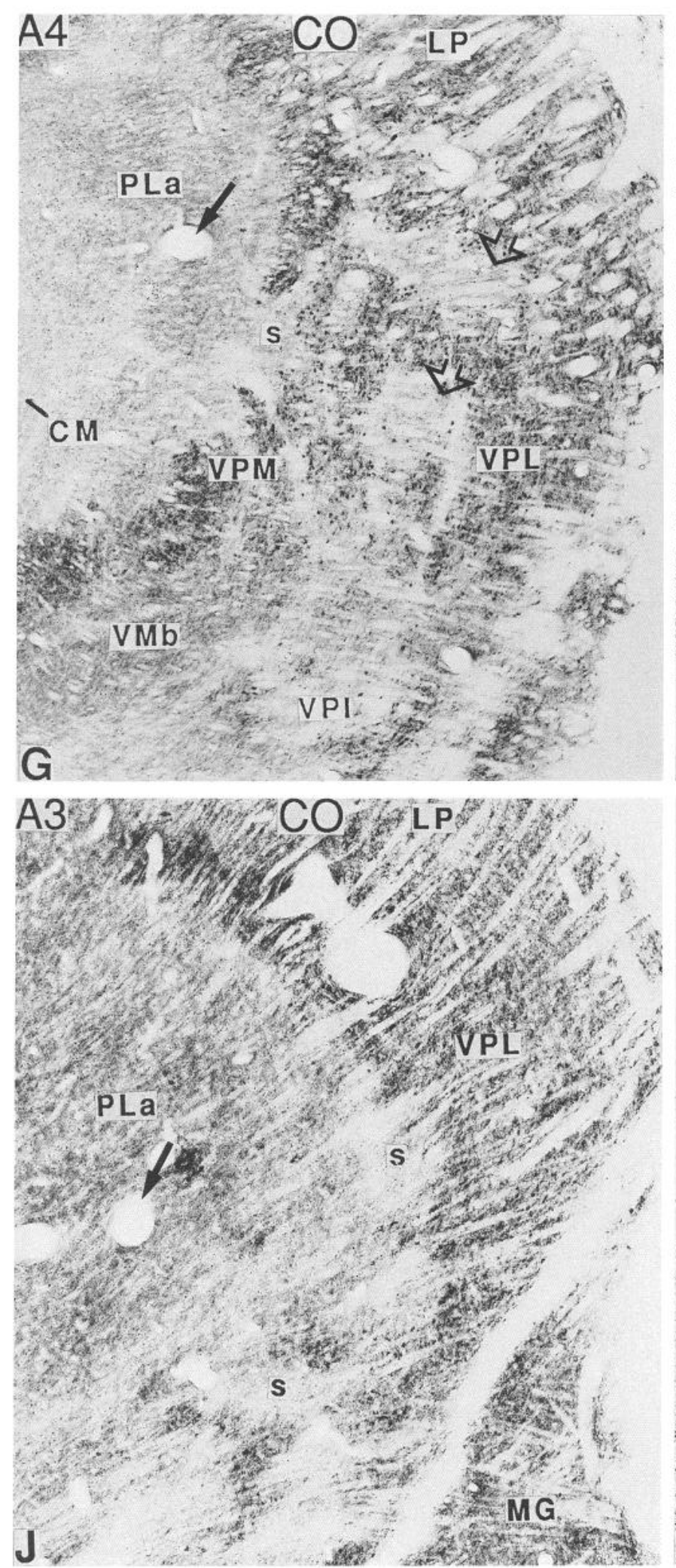

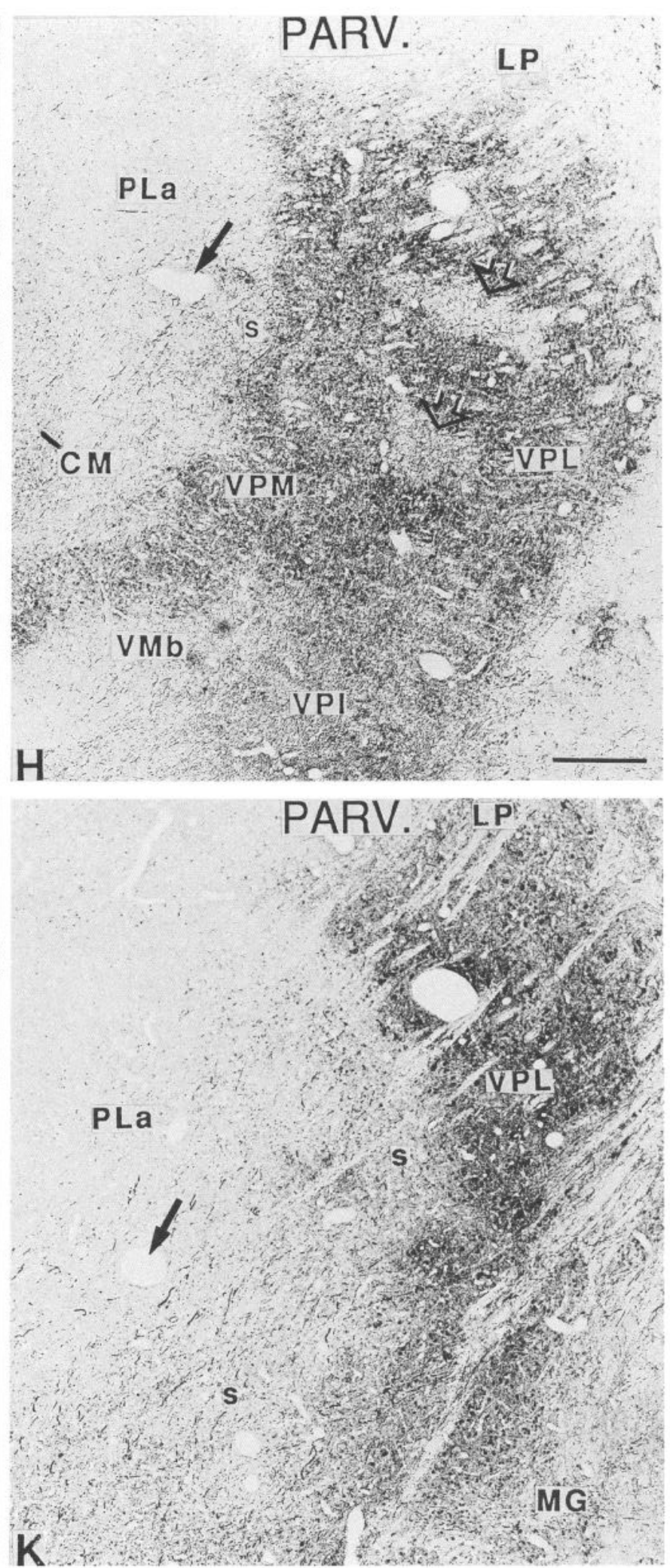

Figure 2. Continued.

parvalbumin antisera in the same dilutions as described above for 24 $\mathrm{hr}$ at $4^{\circ} \mathrm{C}$. Then, the sections were washed and incubated for $2 \mathrm{hr}$ at $4^{\circ} \mathrm{C}$ in a solution containing both FITC-labeled donkey anti-rabbit and RITClabeled donkey anti-sheep secondaries. The sections were washed, immediately mounted, and coverslipped with a 1:3 mixture of glycerol and phosphate buffer. In parallel, additional series of sections were immunostained by the $\mathrm{ABC}$ method using the same procedures described earlier.

In two other monkeys the same approach was used to inject $2-4 \mu \mathrm{l}$ of $20 \%$ horseradish peroxidase (HRP) (Sigma) in normal saline into the anterolateral quadrant of the upper cervical spinal cord. After a survival period of $48 \mathrm{hr}$, the animals were deeply anesthetized and perfused 


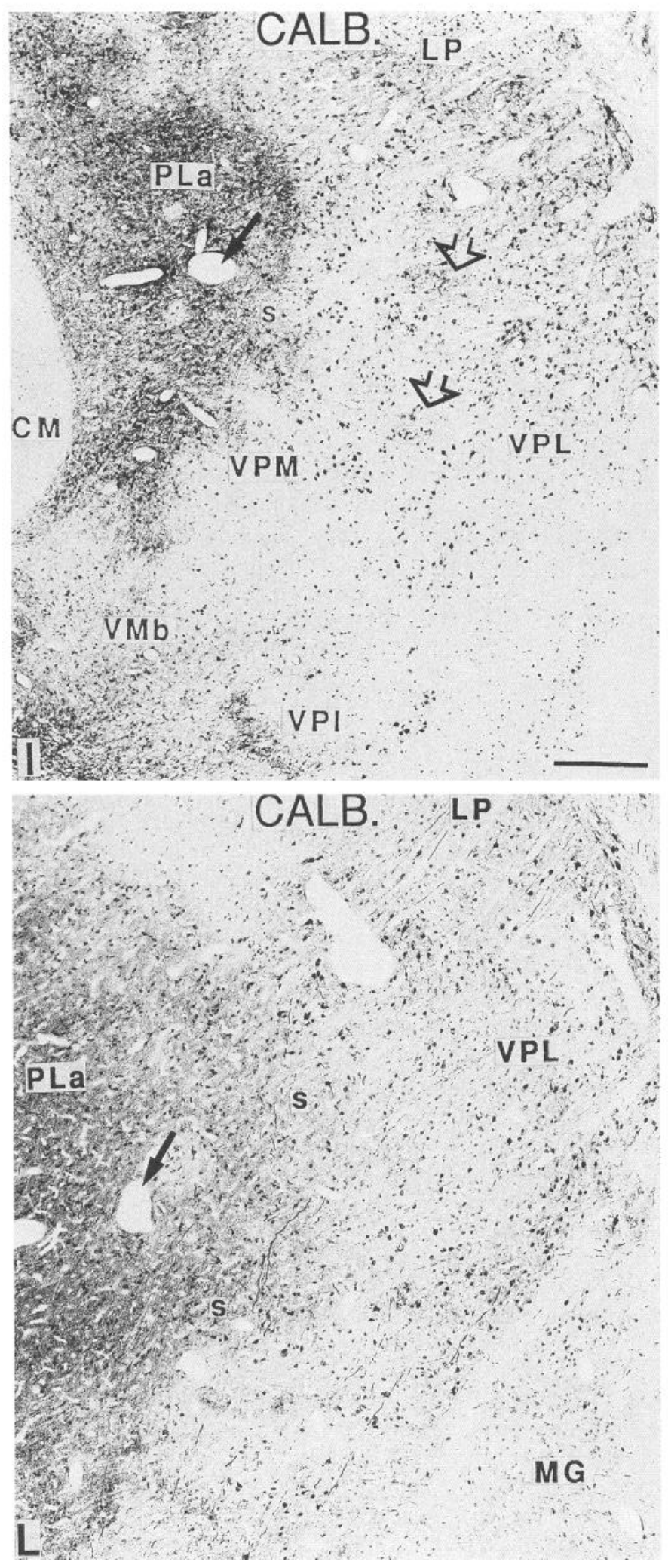

through the heart with a mixture of $1 \%$ paraformaldehyde and $2.5 \%$ glutaraldehyde. The brains and spinal cords were infiltrated with $30 \%$ sucrose in $0.1 \mathrm{M}$ phosphate buffer and then blocked, frozen, and sectioned serially at $50 \mu \mathrm{m}$ on a sliding microtome. In the thalami, two series of frontal sections were stained for HRP using the tetramethylbenzidine reaction (Mesulam, 1978); one of these series was counterstained with thionin. The third series was stained for CO.

The fluorescence material was studied in a Leitz Dialux epifluores- cence microscope equipped with wideband ultraviolet, rhodamine, and fluorescein filter packs. Camera lucida drawings of the sections were made at high and low magnification, and the ramifications of axons anterogradely labeled with Fast Blue and found in the VPL were plotted on them. Camera lucida drawings of the adjacent Nissl-stained sections were then used for outlining the borders of the nucleus, and similar drawings of the distribution of $\mathrm{CO}$ staining in other adjacent sections were later superimposed, using the outlines of the sectioned blood vessels as landmarks.

The material processed for HRP histochemistry was analyzed by light microscopy. Camera lucida drawings were made of serial sections at low magnification, and labeled terminal ramifications were plotted on the drawings from microscopic examination at higher magnification, using blood vessel profiles as guides. Camera lucida drawings were also made of the CO-stained sections, and regions of VPL that showed different intensities of histochemical staining were outlined. The drawings of the sections containing HRP-labeled fibers were then superimposed on those of the CO-stained sections, and correlations were made of the locations of $\mathrm{CO}$ activity and HRP-labeled terminal ramifications.

Retrograde labeling. In four monkeys, bilateral craniotomies were made under intravenous Nembutal anesthesia and aseptic conditions to expose the left and right postcentral gyri or both the pre- and postcentral gyri on each side. On the left side in two animals, penetrations with a tungsten microelectrode were made in the first somatosensory area (SI) in a region corresponding to the middle third of the postcentral gyrus, in order to locate and partially map, by means of multiunit recording, the cortical region containing the representation of the upper limb. Upon identification of a region containing neurons responding to tactile stimulation of the contralateral hand or arm, the recording electrode was replaced by a micropipette with a tip diameter of $20-50 \mu \mathrm{m}$ and filled with $5 \%$ aqueous Fast Blue (Fig. 1). This was lowered to a depth of $1-1.5 \mathrm{~mm}$, and $0.1-0.2 \mu \mathrm{l}$ was injected at this and at two to four surrounding sites by air pressure pulses. On the right side, an approximately corresponding cortical region was located visually, but electrode penetrations were not made in it. The arachnoid mater spanning the central sulcus was incised in this region to permit dye to flow into the central sulcus. An area approximately $1 \mathrm{~cm}^{2}$ was gently rubbed with cotton that had been soaked in $0.1 \mathrm{M}$ acetate buffer $(\mathrm{pH} 3.5)$ until hyperemia was detected at the cortical surface (Fig. 1). Then, a piece of filter paper, previously soaked in 5\% Fast Blue solution, was applied to the cortical surface. After $10 \mathrm{~min}$, it was removed and replaced with a second piece of filter paper also soaked in Fast Blue solution that was left in place. In the other two animals, similar procedures were carried out but the injections and superficial deposits were made in or applied to the adjacent part of the precentral gyri as well. The animals survived for 2 weeks. After that period, they were deeply anesthetized with intravenous Nembutal and perfused with $4 \%$ paraformaldehyde and $0.2 \%$ glutaraldehyde in $0.1 \mathrm{M}$ phosphate buffer. All brains were removed, infiltrated with $30 \%$ sucrose, blocked, frozen in dry ice, and sectioned on a sliding microtome. The cortical blocks containing the injection sites were cut into $30 \mu \mathrm{m}$ sagittal sections and mounted immediately to determine the spread of the injection or superficial application of Fast Blue. An adjacent series was stained with thionin. The thalami from these brains were cut in series of alternating $15 \mu \mathrm{m}$ and $30 \mu \mathrm{m}$ frontal or horizontal sections. The $30 \mu \mathrm{m}$ sections were processed for Nissl staining and $\mathrm{CO}$ histochemistry, while the $15 \mu \mathrm{m}$ sections were processed for single or double immunofluorescence staining for calbindin and/or parvalbumin.

In three monkeys, the identical procedure was followed for exposing and identifying the upper limb representation but HRP was injected and applied instead of Fast Blue. A micropipette with a tip diameter of $20 \mu \mathrm{m}$ and containing a $20 \%$ solution of HRP in normal saline was returned to one of the recording tracks and lowered to a depth of 1.5 $\mathrm{mm}$, and $0.05 \mu \mathrm{l}$ was injected by air pressure. On the homotopic region of the opposite hemisphere, a superficial deposit of HRP was made, using a piece of filter paper soaked in 50\% HRP. The animals survived for $2 \mathrm{~d}$ and were then deeply anesthetized and perfused with $1 \%$ paraformaldehyde and $2.5 \%$ glutaraldehyde in $0.1 \mathrm{M}$ phosphate buffer. The brains were removed, blocked, infiltrated with $30 \%$ sucrose, frozen in dry ice, and sectioned on a sliding microtome. Blocks containing the thalamus and SI were cut into a series of $50 \mu \mathrm{m}$ frontal or sagittal sections. Two out of every four sections were processed by the tetramethylbenzidine method. Every other section of these two was counterstained with thionin. The remaining pair was stained for $\mathrm{CO}$ or with thionin. The outlines of the neurons retrogradely labeled after deep injections and superficial applications of HRP were drawn at high mag- 


\section{219}

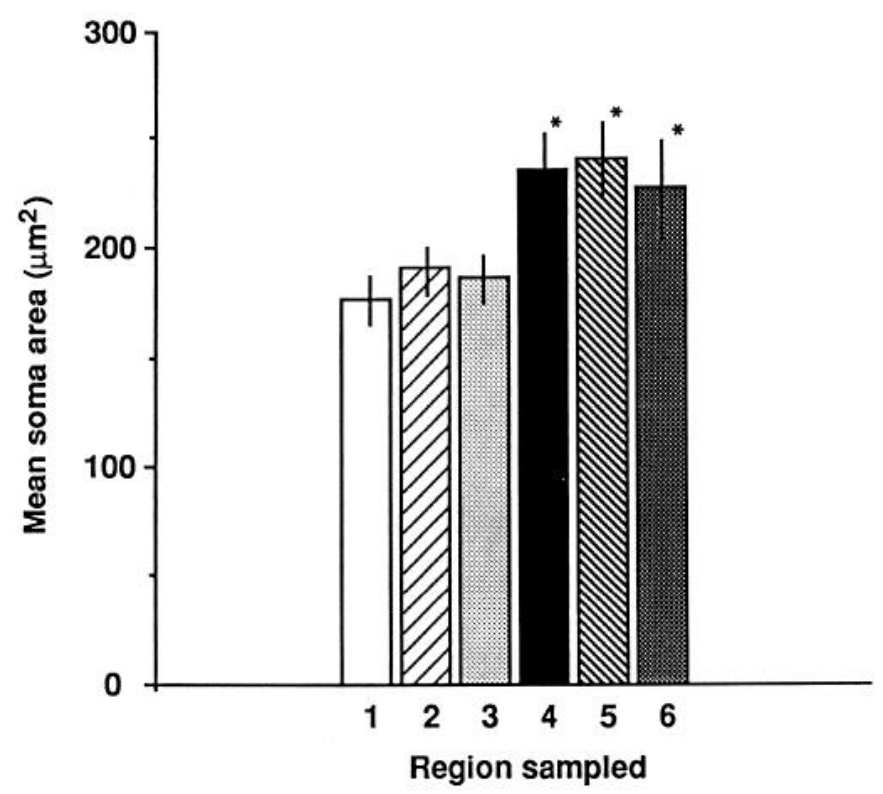

FM 10

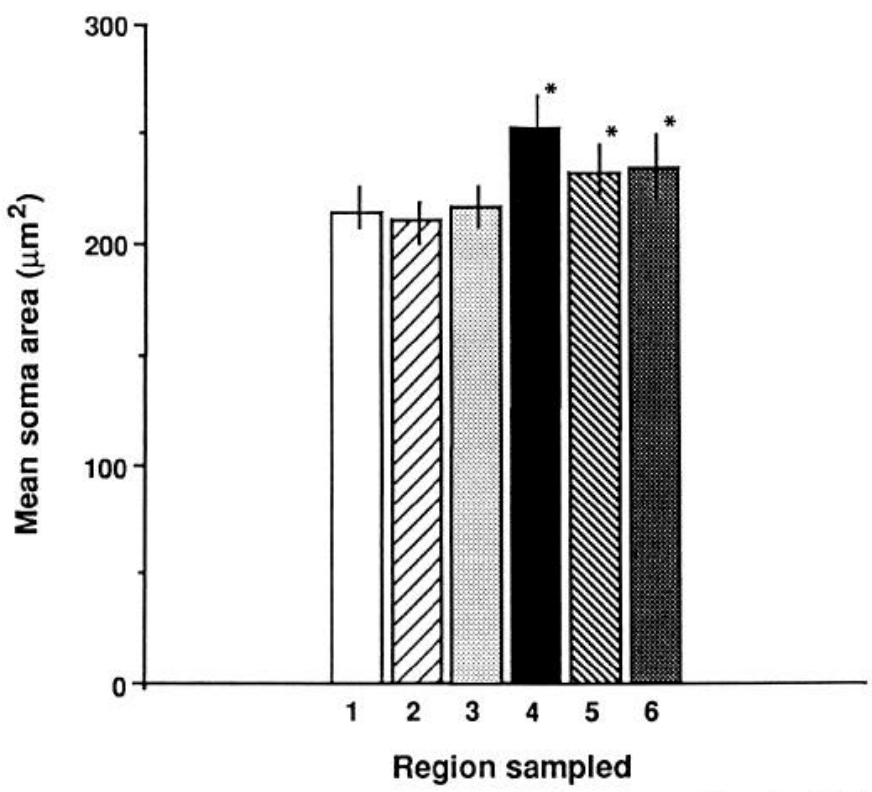

Figure 3. Sizes of somata of calbindin- (1-3) and parvalbumin- (4-6) immunoreactive somata in the core $(1,4)$, medial $(2,5)$, and anterodorsal $(3,6)$ aspects of VPL in two monkeys (CM219, FM10). Calbindin-immunoreactive somata are consistently smaller than parvalbuminimmunoreactive somata. Asterisks indicate that differences are statistically significant $(P<0.001)$.

nification $(100 \times)$ with a camera lucida. Only neurons cut through the nucleus were plotted, and care was taken not to include in the outline the stained initial segments of axons and dendrites. The perimeters and areas of these neurons were measured by means of the digitizing tablet. For each case, the somal areas of thalamocortical neurons labeled by superficial applications of HRP were compared with those of neurons labeled by deep injections, using the nonparametric Kruskal-Wallis and Mann-Whitney $U$ tests.

\section{Results}

The results are presented in three major sections dealing with (1) the relationships of parvalbumin- and calbindin-immuno- reactive cells to compartments of VPL defined by CO staining (Figs. 2, 3; Table 1), (2) the locations of SI-projecting cells in relation to the $\mathrm{CO}$ compartments and the parvalbumin- and calbindin-immunoreactive cells (Figs. 4-10), and (3) the relationships of spinothalamic fiber terminations to the $\mathrm{CO}$ compartments (Figs. 11-14). To avoid duplication of figures, the description of the CO compartments and distributions of parvalbumin- and calbindin-immunoreactive cells will be made by reference to figures that appear in all three major sections of the results (Figs. 2, 4, 5-7, 11, 12).

\section{CO compartments in VPL}

CO staining in VPL is not homogeneous (Figs. $2 A, D, G, J$ ). In the anterior one-third of the nucleus, $\mathrm{CO}$ activity is consistently weaker than in the medially adjacent VPM, and similar in density to that in the moderately to lightly stained ventral posterior inferior nucleus (VPI) and ventral lateral posterior nucleus (VLp). This is well seen in horizontal sections (Fig. 5A). On moving posteriorly, there is an increase in $\mathrm{CO}$ staining that first becomes visible in the medial part of VPL (Fig. $2 A$ ). In VPL, as in VPM, both neuropil and neuronal somata show CO activity. In VPL, the pattern of CO staining is broken by horizontal bundles of unstained fibers that cross the nucleus from medial to lateral, toward the internal capsule, and also by two or more incomplete vertical laminae of absent staining that tend to divide the nucleus into medial and lateral parts. Of these laminae, a medial one is the most distinct and clearly separates VPL from VPM, except for its most dorsal portion where the two nuclei tend to merge (Fig. 2A). A second lamina is commonly present in the middle of the mediolateral extent of VPL (Fig. $2 A, D, G$ ). Apart from the fiber bundles, $\mathrm{CO}$ staining in the anterior third of VPL is relatively evenly distributed. However, even here a number of zones of variable size and length that show enhanced $\mathrm{CO}$ staining alternate with other areas of weaker $\mathrm{CO}$ activity. The contrast between $\mathrm{CO}$-rich and $\mathrm{CO}$-weak zones becomes far more evident in the middle third of VPL (Fig. $2 D$; see also Figs. $4 D$, $5 A$ ). Although the density of the more heavily stained zones never approaches that in VPM, it is significantly heavier than in the anterior third of VPL, so that the contrast between weakly and densely stained zones is enhanced. The staining level of the paler zones is comparable to that in the matrix region (Rausell and Jones, 1991b) of VPM and in the basal ventral medial nucleus $(\mathrm{VMb}$ ) and VPI (Fig. 2D,G).

In the posterior one-third of VPL, the weakly stained areas in the interior of VPL expand and start to merge with one another close to the medial aspect of the nucleus, the unstained fiber lamina separating VPL and VPM breaks down, and the CO-weak zones of VPL also merge with the VPM matrix at the posterior pole of VPM. A CO-weak posterior cap is then defined in the posteromedial aspect of VPL and covers the posterior pole of VPM (Fig. $2 J$; see also Figs. $4 G, 12 F$ ). This lies anteromedial to the anterior pulvinar nucleus (PLa) and merges with the posterior group of nuclei (Po) and with PLa. The PLa nucleus, medial to VPM, shows weak CO staining of a density similar to that in the VPM matrix. The two are incompletely separated by a thin, partial, fiber lamina that is unstained for $\mathrm{CO}$. As the matrix of VPM and the CO-weak zones of VPL expand to form the posterior cap, the unstained lamina breaks down, so that PLa is no longer separated from the cap or from the posterior nuclear region. It can usually be distinguished by a slightly greater density of $\mathrm{CO}$ staining (Fig. $2 J$ ).

After overlapping camera lucida drawings and photomicro- 
Table 1. Densities by area $\left(N_{a}\right)$ and by volume $\left(N_{v}\right)$ of calbindin- and parvalbumin-immunoreactive somata in the core, medial, and anterodorsal parts of VPL of two monkeys (CM219, FM10)

\begin{tabular}{|c|c|c|c|c|c|c|}
\hline & $N$ & $\begin{array}{l}\text { Area sampled } \\
\left(\mathrm{mm}^{2}\right)\end{array}$ & $N_{a}$ & $d(\mu \mathrm{m})$ & $\bar{D}(\mu \mathrm{m})$ & $N_{v}$ \\
\hline \multicolumn{7}{|l|}{ CM219 } \\
\hline \multicolumn{7}{|l|}{ VPL core } \\
\hline Calbindin & 112 & 0.70 & $160.4(38 \%)$ & 7.61 & 9.66 & $842(39.5 \%)$ \\
\hline Parvalbumin & 198 & 0.77 & $257.1(62 \%)$ & 7.9 & 10.03 & $1285(60.5 \%)$ \\
\hline \multicolumn{7}{|l|}{ VPL medial } \\
\hline Calbindin & 143 & 0.70 & $204 \quad(41 \%)$ & 7.12 & 9.04 & $1113(44 \%)$ \\
\hline Parvalbumin & 225 & 0.77 & $292.2(59 \%)$ & 8.29 & 10.5 & $1390(56 \%)$ \\
\hline \multicolumn{7}{|l|}{ VPL ant/dors } \\
\hline Calbindin & 101 & 0.70 & $144.2(41 \%)$ & 7.52 & 9.55 & $758(44 \%)$ \\
\hline Parvalbumin & 157 & 0.77 & $203.8(59 \%)$ & 8.40 & 10.66 & $970(56 \%)$ \\
\hline \multicolumn{7}{|l|}{ FM10 } \\
\hline \multicolumn{7}{|l|}{ VPL core } \\
\hline Calbindin & 96 & 0.77 & $124.6(35 \%)$ & 8.00 & 10.16 & $623(36 \%)$ \\
\hline Parvalbumin & 176 & 0.77 & $228.5(65 \%)$ & 8.30 & 10.54 & $1088(64 \%)$ \\
\hline \multicolumn{7}{|l|}{ VPL medial } \\
\hline Calbindin & 145 & 0.77 & $188.3(45 \%)$ & 8.00 & 10.16 & $941(50 \%)$ \\
\hline Parvalbumin & 151 & 0.77 & $196 \quad(55 \%)$ & 8.50 & 10.79 & $933(50 \%)$ \\
\hline \multicolumn{7}{|l|}{ VPL ant/dors } \\
\hline Calbindin & 108 & 0.77 & $140.2(45 \%)$ & 8.00 & 10.16 & $701(45 \%)$ \\
\hline Parvalbumin & 132 & 0.77 & $171.4(55 \%)$ & 8.20 & 10.40 & $857(55 \%)$ \\
\hline
\end{tabular}

Mean diameter $(\bar{d})$ of the somatic profiles was calculated from $d=\sqrt{4 A / \pi}$, in which $A$ is the area of each protile. Density by volume was calculated by the DeHoff and

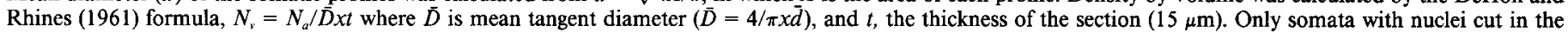
plane of the sections were used. Percentages are those relative to the total immunoreactive population, not to the total population of VPL neurons.

graphs obtained from adjacent Nissl-stained and CO-stained sections, it becomes clear that there is a strong tendency for the CO-weak zones of VPL to correspond to parts of VPL dominated by smaller neurons and for the CO-rich regions to correspond to parts of VPL containing mixed populations of smaller and larger cells (Fig. 4A-H).

\section{Differential distribution of parvalbumin and calbindin}

Parvalbumin. Parvalbumin immunoreactivity is present in large numbers of neuronal somata throughout VPL (Fig. 2; seen also at high magnification in Fig. 10B). The entire nucleus, and especially the posteromedial two-thirds, is traversed by large numbers of parvalbumin-immunoreactive fibers ascending from the medial lemniscus and entering VPL through VPI and VMb (Fig. $2 B$ ). Parvalbumin-positive fibers are also contained within fiber bundles corresponding to the unstained bundles seen in $\mathrm{CO}$ stained preparations (Fig. $2 B, E$ ). Within the nucleus, many irregularly arranged fiber segments and terminal-like boutons are parvalbumin immunoreactive and fill the neuropil between the parvalbumin-immunoreactive cell bodies (Fig. 10B).

Immunoreactivity for parvalbumin is shown in different degrees by VPL neurons. Large neurons tend to be densely stained, whereas the staining of medium- and smaller-sized neurons is variable, and both densely and weakly stained somata can be found among them. The distribution of parvalbumin-immunorcactive ncurons in VPL varies depending on the anteroposterior level and tends to follow the zones of denser CO staining seen in adjacent sections (Fig. $2 D, E, G, H$ ). Groups of densely stained neurons and surrounding fibers tend to be concentrated in ventral and medial regions in anterior parts of the nucleus (Fig. 2B). In the middle third of the nucleus, parvalbumin immunoreactivity is more widespread but is often interrupted by regions that contain fewer parvalbumin-positive fibers and few or no parvalbumin-immunoreactive cells (Fig. $2 E, G$ ). These correspond to $\mathrm{CO}$-weak regions in the adjacent sections (Fig. $2 D, G)$. Close to the posterior pole of the nucleus and conforming to the posterior cap, the dorsal and medial periphery of VPL shows more scattered parvalbumin-immunoreactive cells and fewer immunoreactive fibers than the lateral aspect, the two regions corresponding to zones of weak and dense $\mathrm{CO}$-staining, respectively (Fig. $2 J, K ; 4 G, I$ ). The cap is devoid of parvalbumin-immunoreaclive cells, and any immunoreactive fibers in it are vertical and appear to be ascending through it from the medial lemniscus (Fig. 4I).

Calbindin. Calbindin immunoreactivity is present throughout VPL. Although the calbindin antiserum does not distinguish between $28 \mathrm{kDa}$ calbindin and $29 \mathrm{kDa}$ calretinin, for ease of description, immunostaining with it will be referred to as calbindin immunoreactivity. The density of calbindin immunostaining is considerably less than the density of parvalbumin immunostaining (Fig. 2C,F,I,L; seen also in Figs. 6B, 11). This appearance is due primarily to the virtual absence of calbindin immunoreactivity in fibers in the nucleus, but there also appears to be significantly fewer calbindin cell somata. (This is confirmed quantitatively below.) Mainly medium- and small-sized neurons are stained for calbindin, but occasional large cells are also stained (Fig. 10D,F). A few calbindin-immunoreactive fibers can be seen leaving VPL and entering the reticular nucleus. However, no calbindin-positive fibers can be seen entering VPL from the medial lemniscus.

When comparing Nissl-stained, CO-stained, and parvalbumin- or calbindin-immunostained sections, there is a slight tendency for calbindin immunoreactivity in VPL to occur in a pattern complementary to that of dense $\mathrm{CO}$ staining and par- 

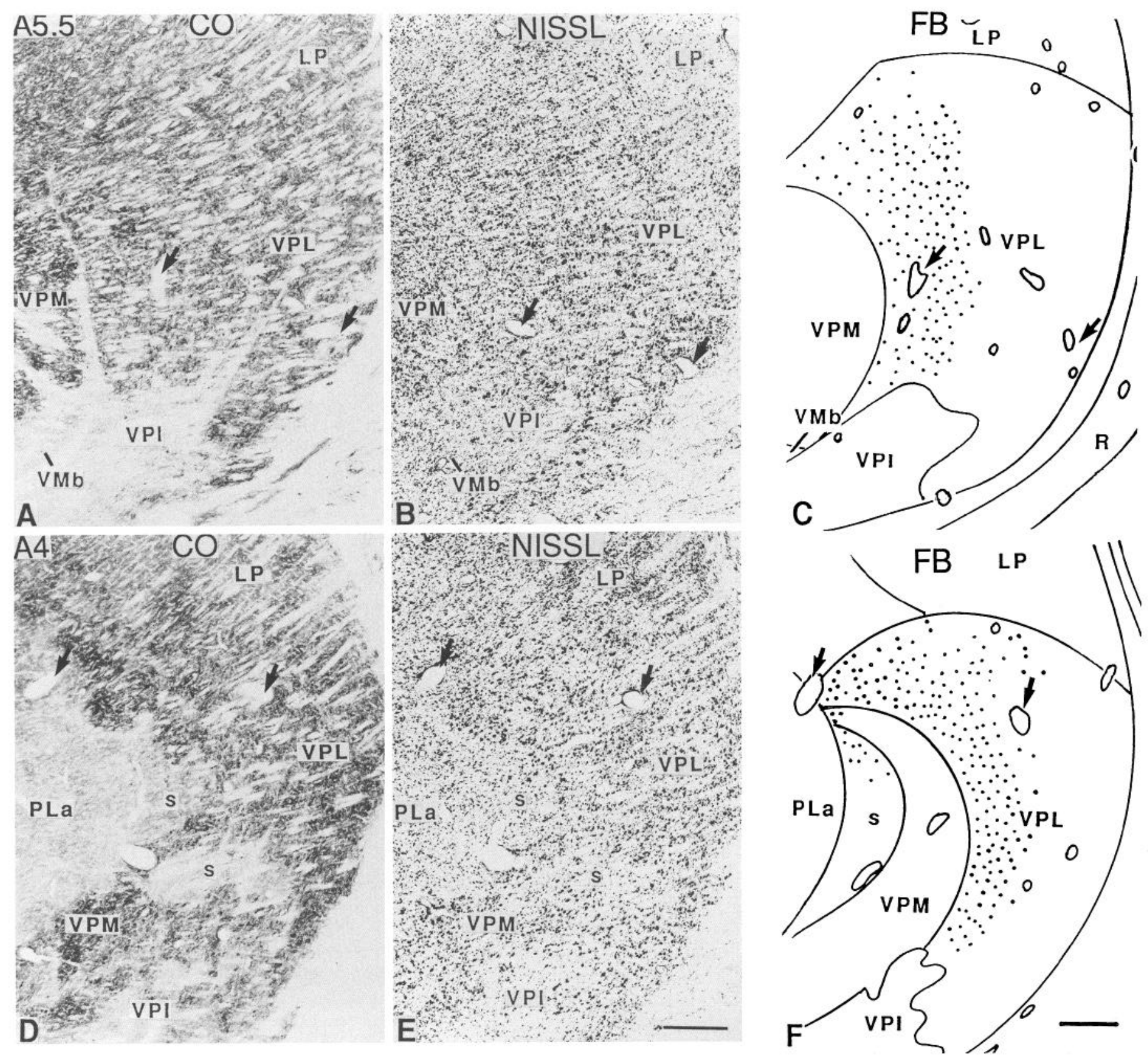

valbumin immunoreactivity (Fig. 2). In sagittal sections, in particular (Fig. 11), calbindin-immunoreactive cells appear more concentrated in the anterodorsal aspect of VPL (Fig. 11), forming a shell that curves medially to merge with the matrix zone of VPM, ventrally to merge with VPI, and posteriorly to merge with the VPL/VPM cap and with PLa, Po, and the suprageniculate nucleus (SG). That is, the region of denser calbindin staining tends to be continuous with regions of VPM and with other nuclei that contain high densities of small cells and show weak $\mathrm{CO}$ staining, high calbindin immunoreactivity, and weak or absent parvalbumin immunoreactivity (Figs. 2, 4, 5). Elsewhere in VPL, small groups of isolated, calbindin-positive cells are widely scattered (Figs. 2, 5, 11). No part of the nucleus is completely free of calbindin cells, but the absence of parvalbuminimmunoreactive cells from the $\mathrm{CO}$-weak zones ensures that these zones are dominated by calbindin cells (Fig. $2 G-I$ ).
Colocalization. The majority of cells identified in sections stained by indirect immunofluorescence for calbindin and parvalbumin were stained for one antigen only, confirming data previously reported in detail by Jones and Hendry (1989). An occasional cell among the larger neurons in regions corresponding to the CO-dense and parvalbumin-rich zones, however, was immunoreactive for both antigens.

Sizes and densities of parvalbumin and calbindin cells. Figure 3 shows the mean areas of calbindin- and parvalbumin-immunoreactive somata sampled from the core of VPL and from its lateral and medial aspects in two animals. In each of the three regions of each animal, calbindin cells are consistently smaller than parvalbumin cells $(p<0.001)$, but there is no difference in the sizes of calbindin cells when different regions of the same nuclei are compared $(p<0.9)$. Statistical testing by multiple ANOVA indicated that all neurons in one animal 

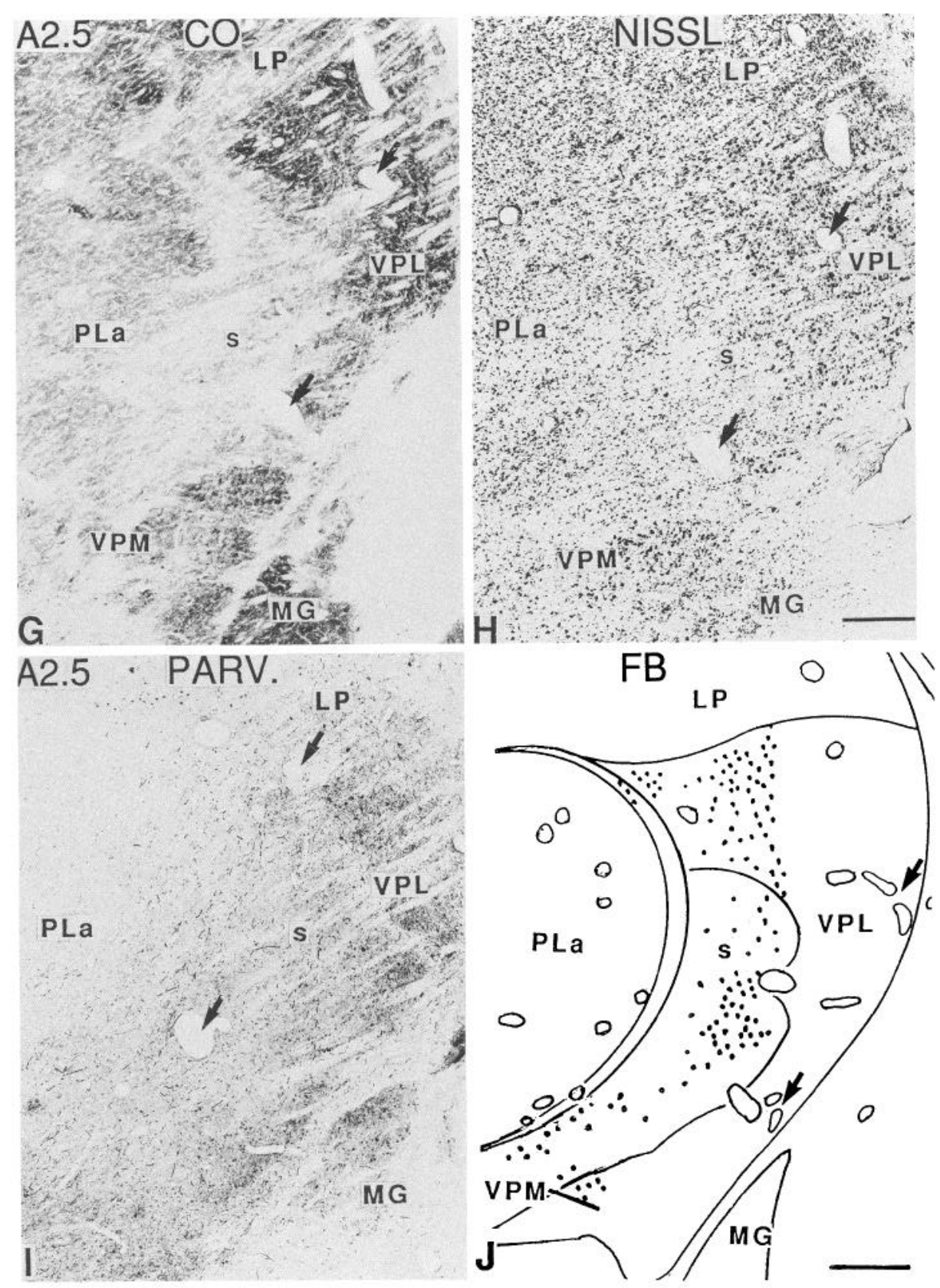

Figure 4. Pairs $(A, B ; D, E ; G, H)$ of adjacent $\mathrm{CO}$ - and Nissl-stained frontal sections at anterior, middle, and posterior levels of VPL, matched to camera lucida drawings of a third closely adjacent section from the same brain on which are plotted the distributions of cells retrogradely labeled as the result of injecting FB into layers I-V of the SI hand representation $(C, F, J) . I$, stained immunocytochemically for parvalbumin, is adjacent to section from which $J$ was drawn. Arrows indicate same blood vessels. Note presence of small, Nissl-stained somata in CO-weak zones $(s)$, correspondence of CO-weak, smallcelled, posterior VPL/VPM cap in $G$ and $H$ with lack of parvalbumin-immunoreactive somata $(I)$, and presence of retrogradely labeled cells in both $\mathrm{CO}$ weak and CO-rich compartments. For abbreviations, see Appendix. Scale bars, $1 \mathrm{~mm}(E$ also for $A, B$, and $D ; F$ also for $C ; H$ also for $G ; J$ also for $I)$. were slightly larger than those of the second and that this difference approached statistical significance $(F=8.046 ; P=$ 0.0046 ). However, the sizes of neurons stained for calbindin or parvalbumin showed far greater differences that were highly significant statistically $(F=38.441 ; P=0.0001)$ and could not be accounted for on the basis of interanimal variability. Crosscomparisons of sizes of cells stained for a particular calciumbinding protein in different regions $(P=0.8373)$, of animal versus calcium-binding protein $(P=0.0477)$, of animal versus region $(P=0.3562)$, of calcium-binding protein versus region $(P=0.2966)$, and of animal versus protein versus region $(P=$ 0.9066 ) showed that somal size differences are not affected by the region of VPL sampled.

The density of calbindin neurons tended to be lower than that of parvalbumin neurons (Table 1), the calbindin cells, with one exception forming less than $45 \%$ by volume of the total popu- lation of neurons immunoreactive for the two proteins in a given region of the nucleus. There was a consistent trend in the difference between densities of calbindin cells from region to region. In both animals, density by volume was lower (36\% and $39 \%)$ in the core than in the medial (50\% and $44 \%)$ and anterodorsal (45\% and $44 \%$ ) aspects of VPL.

\section{Laminar projections to SI: FB and HRP labeling}

After injections of the tracers that involve all layers of SI, retrogradely labeled neurons are found in a lamella in the medial aspect of VPL and extend dorsoventrally and anteroposteriorly through the nucleus in a region corresponding to the upper limb representation (Fig. 4). Large, medium, and small somata are labeled (Fig. 10C). Superimposition of camera lucida plottings of cells and photomicrographs of CO- and Nissl-stained sections reveals that the neurons occupy both $\mathrm{CO}$-rich and $\mathrm{CO}$-weak 

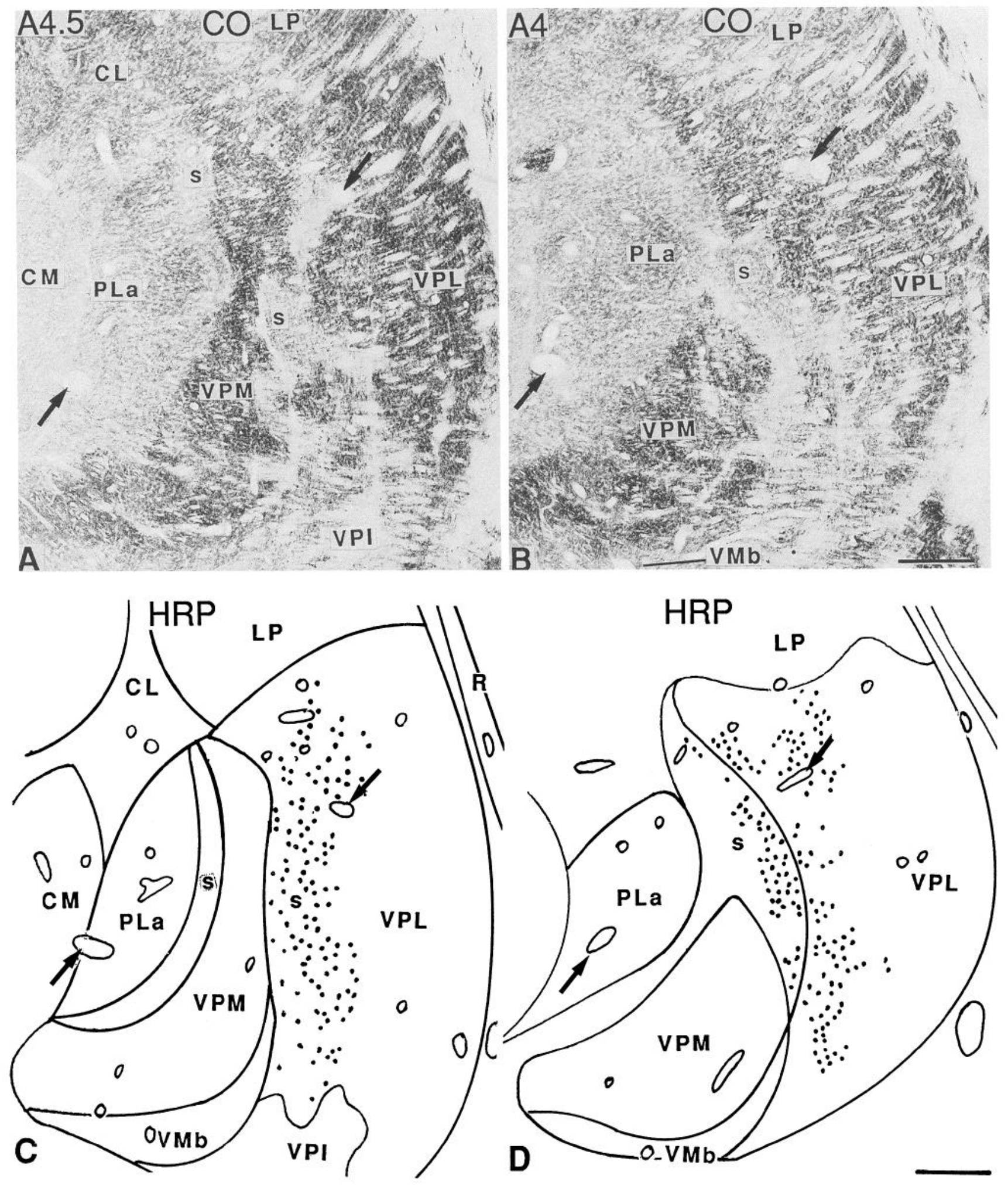

Figure 5. Frontal sections $(A, B)$ stained for $\mathrm{CO}$ activity matched to camera lucida drawings of adjacent sections $(C, D)$ showing the distribution of cells retrogradely labeled with HRP after placement of the material on the surface of SI in the upper limb region. Labeled cells are found particularly, although not exclusively, in the CO-weak $(s)$ zones. For abbreviatons, see Appendix. Arrows indicate same blood vessels. Scale bars, $1 \mathrm{~mm}$.

parts of VPL, and include small labeled neurons in the cap on the posterior surface of VPM (Fig. 4). After superficial applications of FB or HRP confined to layer I or layers I and II of SI, retrogradely labeled neurons are found only in VPL and the posterior cap (Fig. 5). After applications affecting the surface of the precentral gyrus as well, additional labeling is seen in VLp (Fig. 6). Superficial applications of tracer tend to label small neurons in dorsomedial and ventroposterior parts of VPL (and in dorsolateral parts of VLp when the precentral gyrus is affected). Camera lucida drawings of sections through VPL show- 

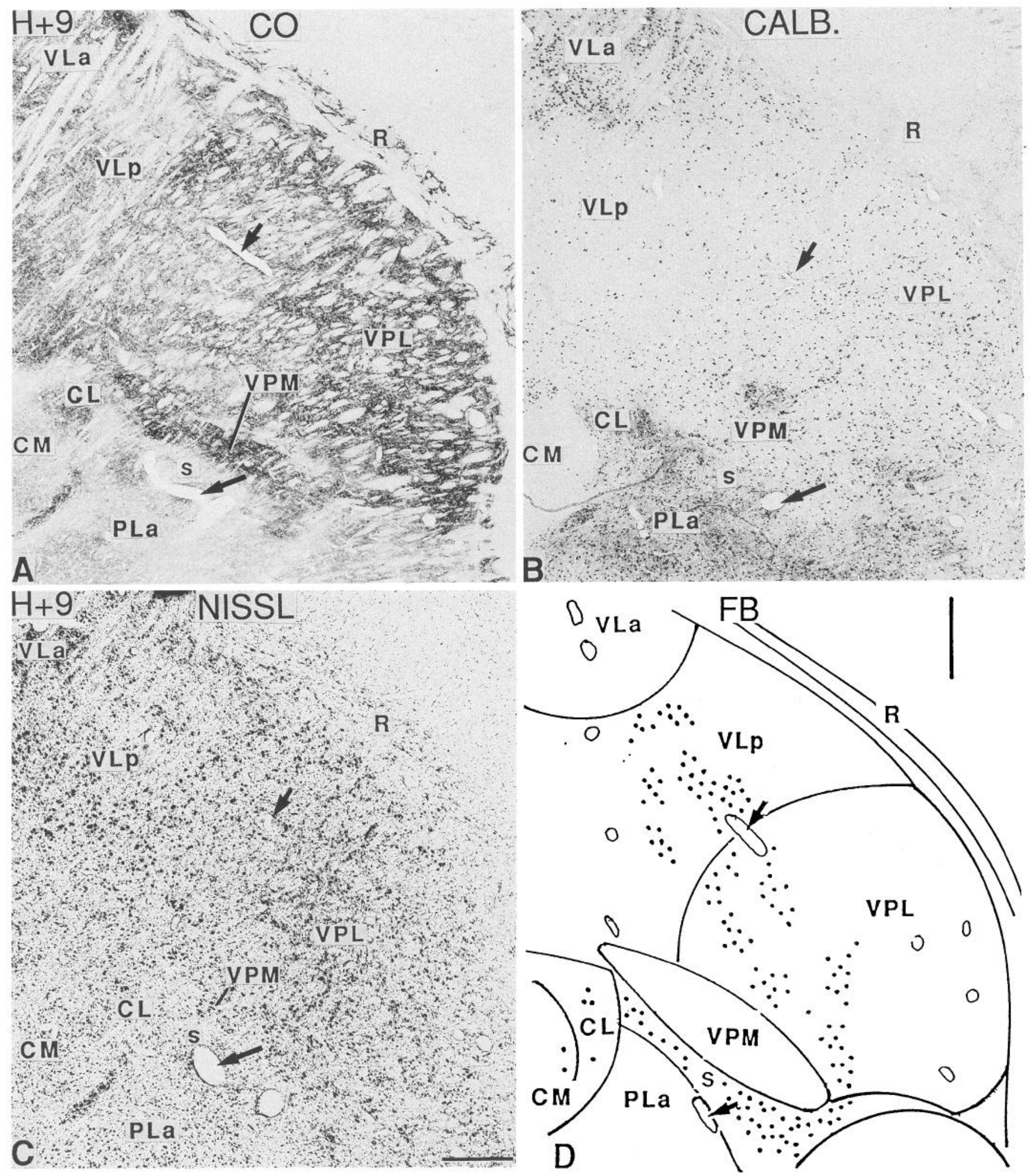

Figure 6. A trio of adjacent horizontal sections through VPL at approximate stereotaxic level +9 , stained for CO activity $(A)$, for calbindin immunoreactivity $(B)$, and with thionin $(C)$, and matched to a camera lucida tracing $(D)$ of a fourth section on which cells retrogradely labeled following placement of FB on the surfaces of the pre- and postcentral gyri, are plotted. Arrows indicate same blood vessels. Note correspondence of small-celled regions with CO-weak anterodorsal part of VPL and presence of retrogradely labeled cells in this region and in small-celled region $(s)$ adjacent to VPM and PLa nuclei. For abbreviatons, see Appendix. Scale bars, $1 \mathrm{~mm}$. 
Figure 7. Enlarged view of part of $\mathrm{CO}$ stained section $(A)$ shown in Figure $5 A$, matched to a photomicrograph of the same region of the uncounterstained, adjacent section containing HRP-labeled cells. Note presence of labeled cells in regions corresponding to $\mathrm{CO}$-weak zones $(s)$. Arrows indicate same blood vessels. For abbreviatons, see Appendix. Scale bar, $500 \mu \mathrm{m}$.
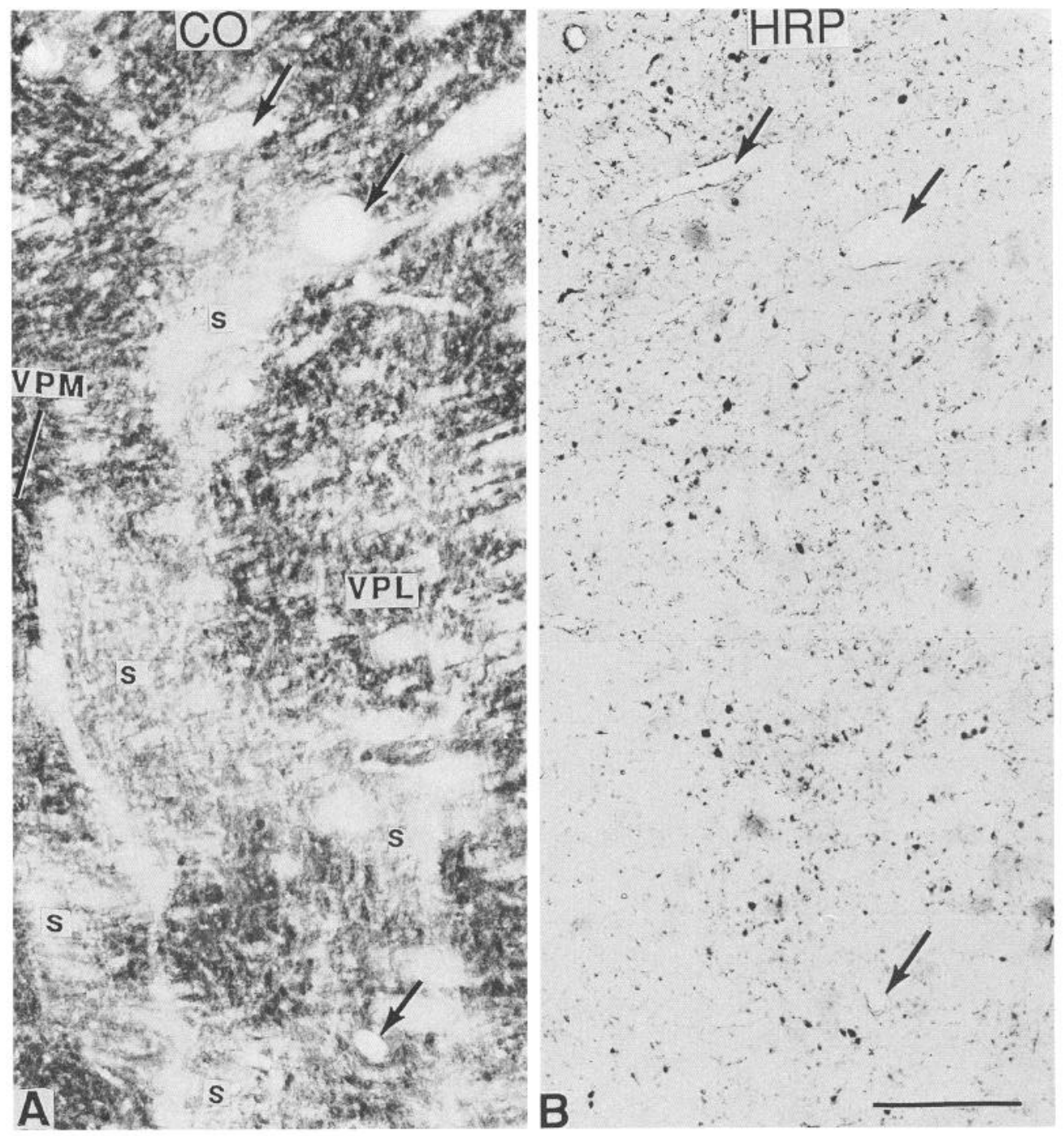

ing the distribution of retrogradely labeled neurons after superficial applications of tracers, when superimposed on photomicrographs of CO- and Nissl-stained sections, reveal that the labeled neurons are found in regions of both dense and weak CO activity (Figs. 5-7). Many, however, are concentrated anterodorsally and posteroventrally in VPL where CO activity is weak and especially in the CO-weak posterior cap (Fig. 6). When found at the center of VPL, the retrogradely labeled, superficially projecting neurons are situated in $\mathrm{CO}$-weak and $\mathrm{CO}$-dense zones but tend to be concentrated in zones of small cells and weaker CO staining (Fig. 7).

The somal sizes of thalamocortical neurons retrogradely labeled after HRP injections affecting layers I-V of SI showed a wider range than those of thalamocortical neurons retrogradely labeled after superficial deposits on the surface of SI (Fig. 8). The mean somal area of $70 \%$ of the population of neurons labeled after injections involving layers I-V was contained within the range of $200-400 \mu \mathrm{m}^{2}$. The mean somal area of $70 \%$ of the neurons labeled after superficial deposits was contained within the range of $100-250 \mu \mathrm{m}^{2}$. The difference between the area values of the two populations was statistically highly significant ( $p<0.0001$, Mann-Whitney $U$ test).

Most of the neurons retrogradely labeled after superficial placements of FB (Fig. 9) are small (15 $\mu \mathrm{m}$ in diameter) and in double-labeled preparations $80-85 \%$ are also immunoreactive for calbindin (Fig. 10E,F). The remaining 15-20\% of the cells labeled after superficial placements of FB are not immunoreactive for calbindin. These are typically small and located at the posterior pole of VPL where its small-celled, CO-weak zones merge with the cap on the posterior surface of VPM. No superficially projecting cells are immunoreactive for parvalbumin. Injections of FB involving layers I-V of SI typically label both smaller calbindin-immunoreactive and medium- and larger-sized parvalbumin-immunoreactive neurons (Fig. 10C,D).

\section{Spinothalamic terminations: $F B$ and HRP labeling}

Terminal ramifications of spinothalamic tract fibers, anterogradely labeled with FB or HRP, are found in the VPL and in parts of adjoining nuclei, especially VLp, the central lateral nucleus (CL), VPI, and Po but with extension into the small-celled matrix of VPM and into parts of PLa and SG (Figs. 11, 12). The labeled terminal ramifications are concentrated in small focal patches $200-500 \mu \mathrm{m}$ in diameter in all of these nuclei. When photographs and camera lucida plottings of the distributions of these labeled terminal ramifications in VPL are superimposed on photomicrographs showing the pattern of his- 


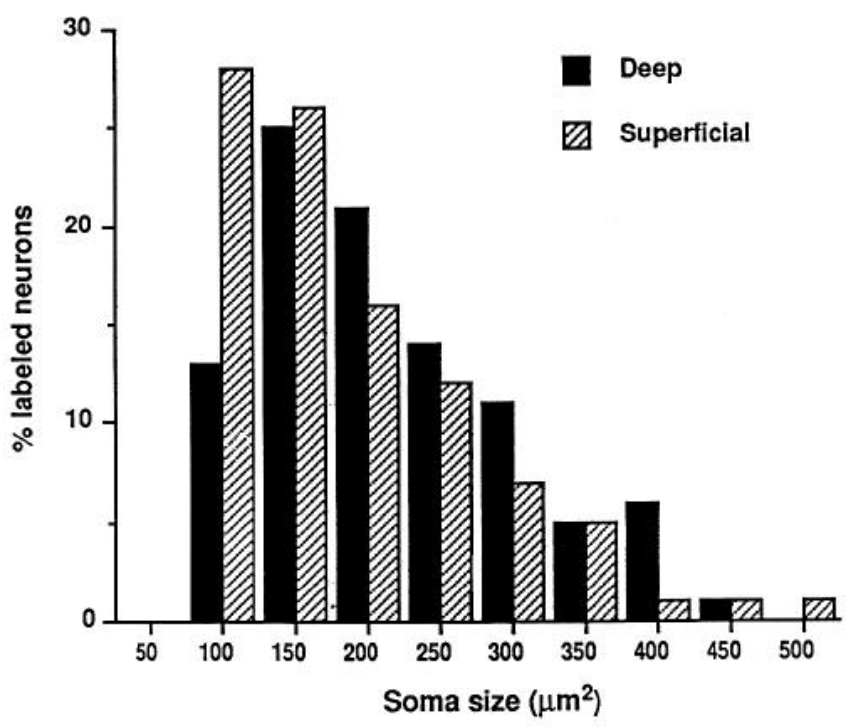

Figure 8. Percentages of neurons with different somal areas taken from corresponding regions of the left and right VPL nuclei in which HRP was injected into layers I-V of the SI upper limb representation on one side (Deep) and placed on the surface of the homotopical region of the opposite side (Superficial). Cells labeled from superficial placements of tracer include a greater number of those with smaller somal sizes.

tochemical staining for $\mathrm{CO}$, a general tendency can be discerned for the spinothalamic terminations to cluster in anterodorsal and posteromedial aspects of VPL, particularly in regions where $\mathrm{CO}$ activity is weakest. This is clearly evident in the posterior VPL/VPM cap (Fig. 13). Patches of terminations in the core of VPL commonly, although not invariably, tend to associate with zones of weak CO staining (Fig. 14A,B). In the adjoining nuclei, especially VPI, Po, PLa, and the small-celled matrix of VPM, the patches of labeled spinothalamic terminations are very clearly associated with nuclei or parts of nuclei that show weak $\mathrm{CO}$ activity overall and high densities of calbindin cells. The use of high concentrations of glutaraldehyde in the HRP fixative precluded direct immunocytochemical staining for parvalbumin and calbindin in the HRP studies. In the FB experiments (Fig. 11) where the fixative permitted immunostaining of sections containing FB-labeled fiber terminations for calbindin and/or parvalbumin immunofluorescence, no spinothalamic fibers labeled with FB could be seen to be immunoreactive for calbindin or parvalbumin (Fig. 14C,D).

\section{Discussion}

This investigation set out (1) to describe the distribution patterns of relay neurons that differentially express the calcium-binding proteins calbindin or parvalbumin in and around the monkey VPL thalamic nucleus and (2) to determine to what extent these cells show connectional relationships with the somatosensory cortex and subcortical afferent pathways that are similar to those established previously for neurons in the VPM. Certain basic principles defined for VPM (Jones et al., 1986a,b; Rausell and Jones, 1991a,b) appear to hold for VPL, but the clear compartmental organization of VPM is far less evident in VPL. Principles that seem to hold for both VPM and VPL are (1) the projection of calbindin-immunoreactive cells to the most superficial layer or layers of the cerebral cortex and of parvalbumin-immunoreactive cells to deeper layers (the nature of deeper injection placements precludes ruling out that calbindin cells

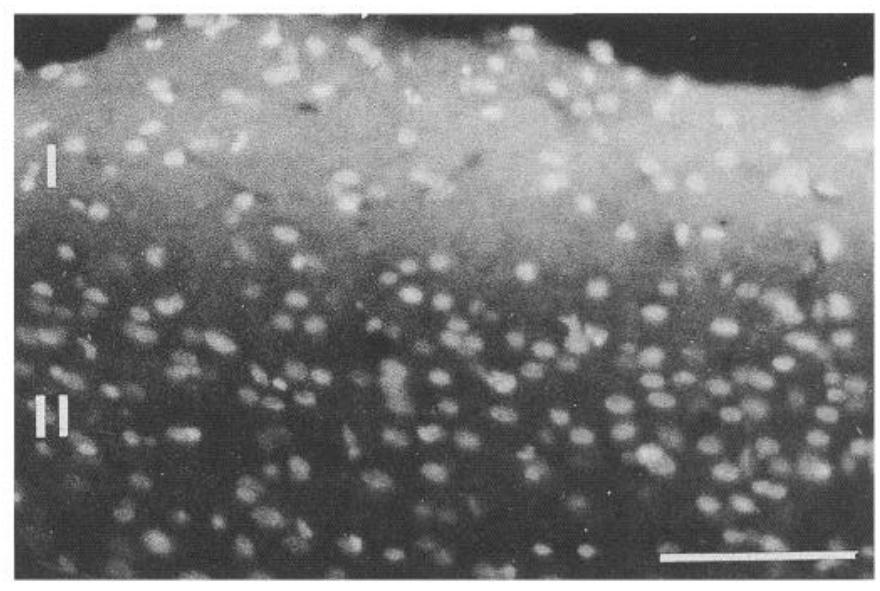

Figure 9. Photomicrograph of part of the surface of SI showing the maximal extent of penetration of the dye following application of FB to the hyperemic surface. Cells labeled in layer II have probably taken up label via dendrites in layer I. Scale bar, $50 \mu \mathrm{m}$.

project to deeper layers as well as to layer I), (2) the consistently smaller size of calbindin-immunoreactive cells in comparison with parvalbumin-immunoreactive cells, and (3) the association of parvalbumin-immunoreactive cells and parvalbumin-immunoreactive afferent fiber terminations with $\mathrm{CO}$-rich domains.

One principle established in VPM that clearly does not hold in VPL is the segregation of calbindin cells in a CO-weak compartment or matrix, although there are certain zones of VPL in which $\mathrm{CO}$-weak zones and calbindin cells coincide and from which parvalbumin cells are excluded. In both nuclei there is a strong tendency for caudal trigeminothalamic or spinothalamic terminations to be concentrated in the $\mathrm{CO}$-weak, calbindin-rich matrix or posterior cap of VPM and in CO-weak zones of VPL, but in both subnuclei, especially VPL, there is a good deal of overlap into the CO-rich, parvalbumin-rich domain.

Intense $\mathrm{CO}$ activity is a feature of the VPL and VPM in other species (Wiener et al., 1987) as well as primates. The association of CO-dense staining patterns in the ventral posterior complex not only with the "lemniscal" (dorsal column and principal trigeminal) system of afferent fibers but also with parvalbumin immunoreactivity in these afferents and in thalamic relay cells, is established by the following observations. Lemniscal fibers terminate in dense clumps that in VPM, at least, are coextensive with the CO-rich rods (Jones et al., 1986a,b). It is undoubtedly the high density of mitochondria in the lemniscal terminals (Ralston, 1969) that results in the intensity of CO activity in zones in which these terminals are concentrated (Wong-Riley, 1979). The lemniscal fibers are parvalbumin positive; this is revealed by direct immunostaining in the present and previous studies (Jones and Hendry, 1989) and by the loss of parvalbumin immunoreactivity and of $\mathrm{CO}$ activity that occurs in the medial lemniscus and in the forelimb representation of VPL following transneuronal degeneration of parvalbumin-immunoreactive relay cells in the cuneate nucleus caused by spinal dorsal rhizotomies (Rausell et al., 1992). Afferent-tracing studies reveal a clear association of trigeminal lemniscal terminations in VPM with CO-rich rods that contain concentrated parvalbumin- and GABA-immunoreactive cells but few or no calbindin-immunoreactive cells (Rausell and Jones, 1991a,b). The situation is less clear in VPL. Bulk labeling of lemniscal terminations by autoradiography or other methods shows an evident clustering pattern (cf., e.g., Fig. $12 A$ of Asanuma et al., 1983, or Fig. 11 

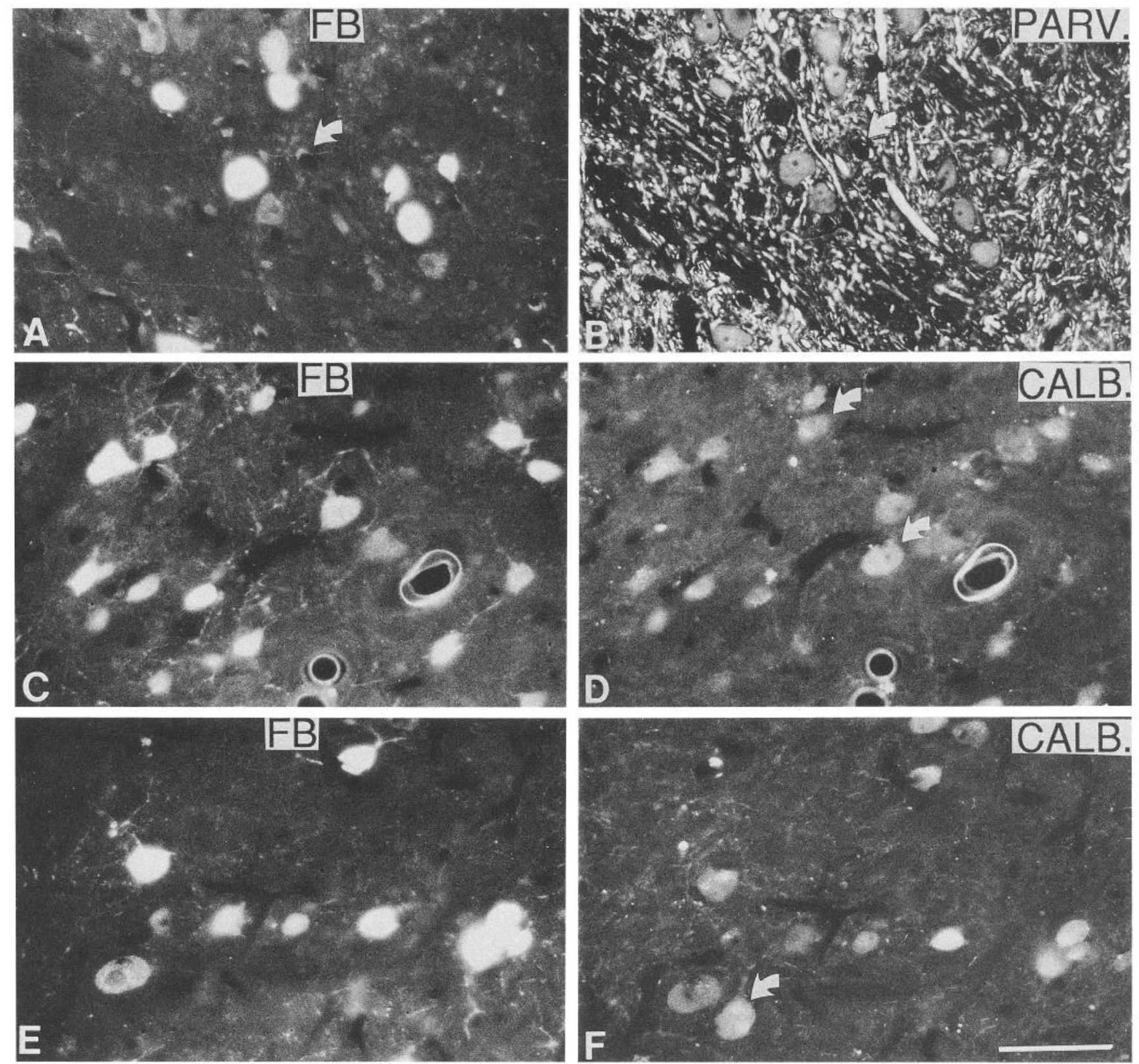

Figure 10. Pairs of fluorescence micrographs, each pair from the same microscopic field. $A$, Retrograde labeling with FB of neurons in VPL following an injection of SI affecting layers I-V. All of these neurons are immunoreactive for parvalbumin $(B)$. Arrows indicate same blood vessel. Note heavy parvalbumin immunoreactivity in fibers. $C$, Retrograde labeling of cells in VPL following injection of FB into layers I-V. Many, but not all $(D$, arrows), of these neurons are also immunoreactive for calbindin in $D$. $E$, Retrograde labeling of neurons in the posterior cap of VPL/ VPM in an animal in which FB was applied to the surface of SI. Most, but not all $(F$, arrow), of these neurons are immunoreactive for calbindin in $F$. Scale bar, $25 \mu \mathrm{m}$.

of Jones et al., 1982). Up to this time, however, these clusters have not been correlated with the CO-enriched zones of VPL. A reexamination of material produced in the past in this laboratory indicates that a considerable number of lemniscal fibers passes through the posterior, CO-weak cap of VPL/VPM en route to VPL but that there are few or no terminal ramifications there. This is evident also in Figures $2 K$ and $4 I$ of this article. The CO-weak, small-celled, calbindin-positive cap receives strong terminations of spinothalamic fibers. In it, therefore, there is a clear dissociation of lemniscal and spinothalamic terminations akin to that seen in the matrix of VPM with which the cap is continuous.
A similar, clear dissociation of lemniscal and spinothalamic terminations is not evident in the remainder of VPL. Although there is some tendency for spinothalamic terminations to cluster in CO-weak zones that contain only calbindin cells, this is by no means an absolute finding. Further investigations involving strategies to label both lemniscal and spinothalamic terminations contemporaneously will be necessary to resolve the issue. The most obvious correlation between the terminations of the spinothalamic tract and the small-celled, CO-weak, calbindinrich domain is in the posterior cap where the matrix of VPM and the small-celled zones of VPL converge.

The general distribution of spinothalamic tract terminations 

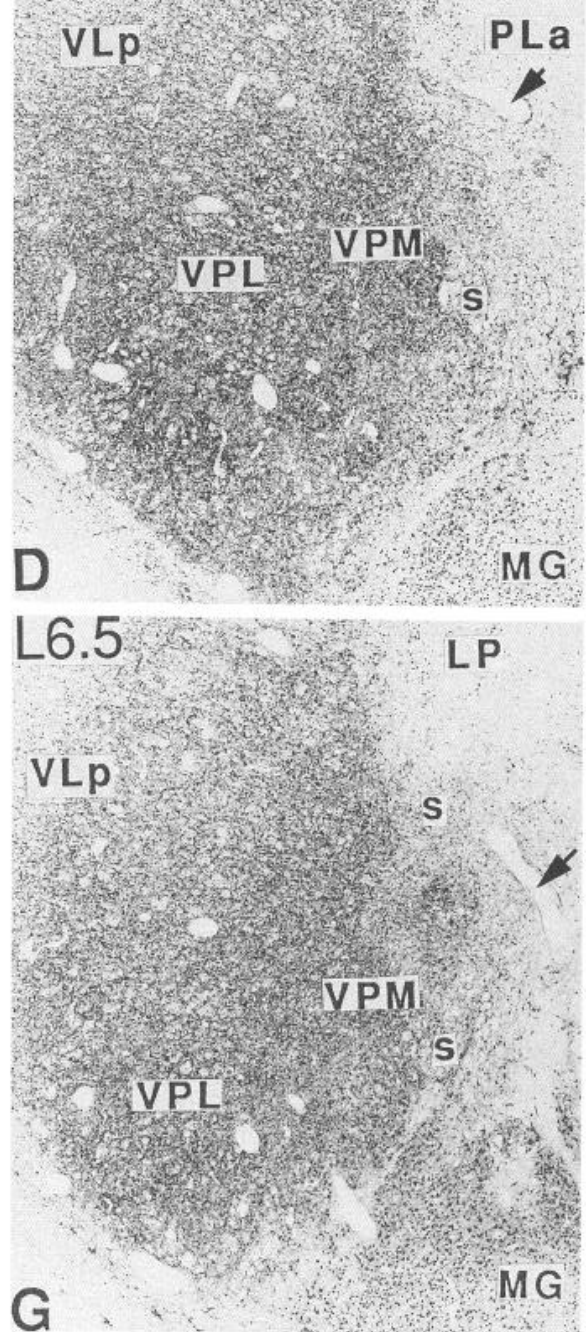
B
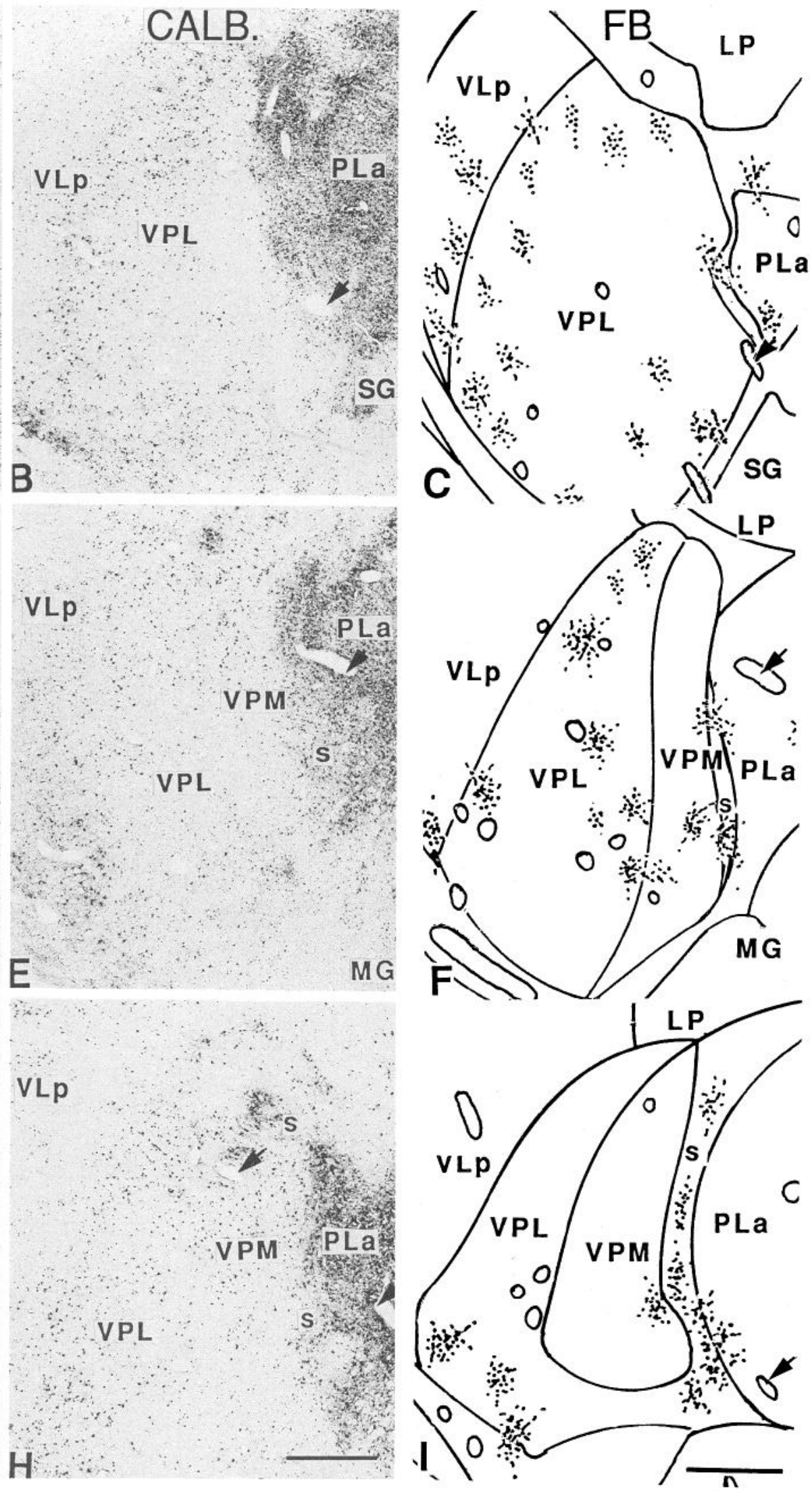

Figure 11. Pairs of adjacent sagittal sections at approximate stereotaxic levels indicated, stained immunocytochemically for parvalbumin (left column) or for calbindin (middle column) and matched to camera lucida drawings of a third adjacent section (right column) on which are plotted the distributions of terminal ramifications of spinothalamic tract fibers anterogradely labeled with FB following injections in the second cervical spinal segment. Arrows indicate same blood vessels. Note somewhat higher density of calbindin-immunoreactive cells in anterodorsal aspect of VPL and tendency for spinothalamic terminations to cluster there and in small-celled $(s)$ cap at posterior pole of VPM. For abbreviatons, see
Appendix. Scale bars, $1 \mathrm{~mm}$. 

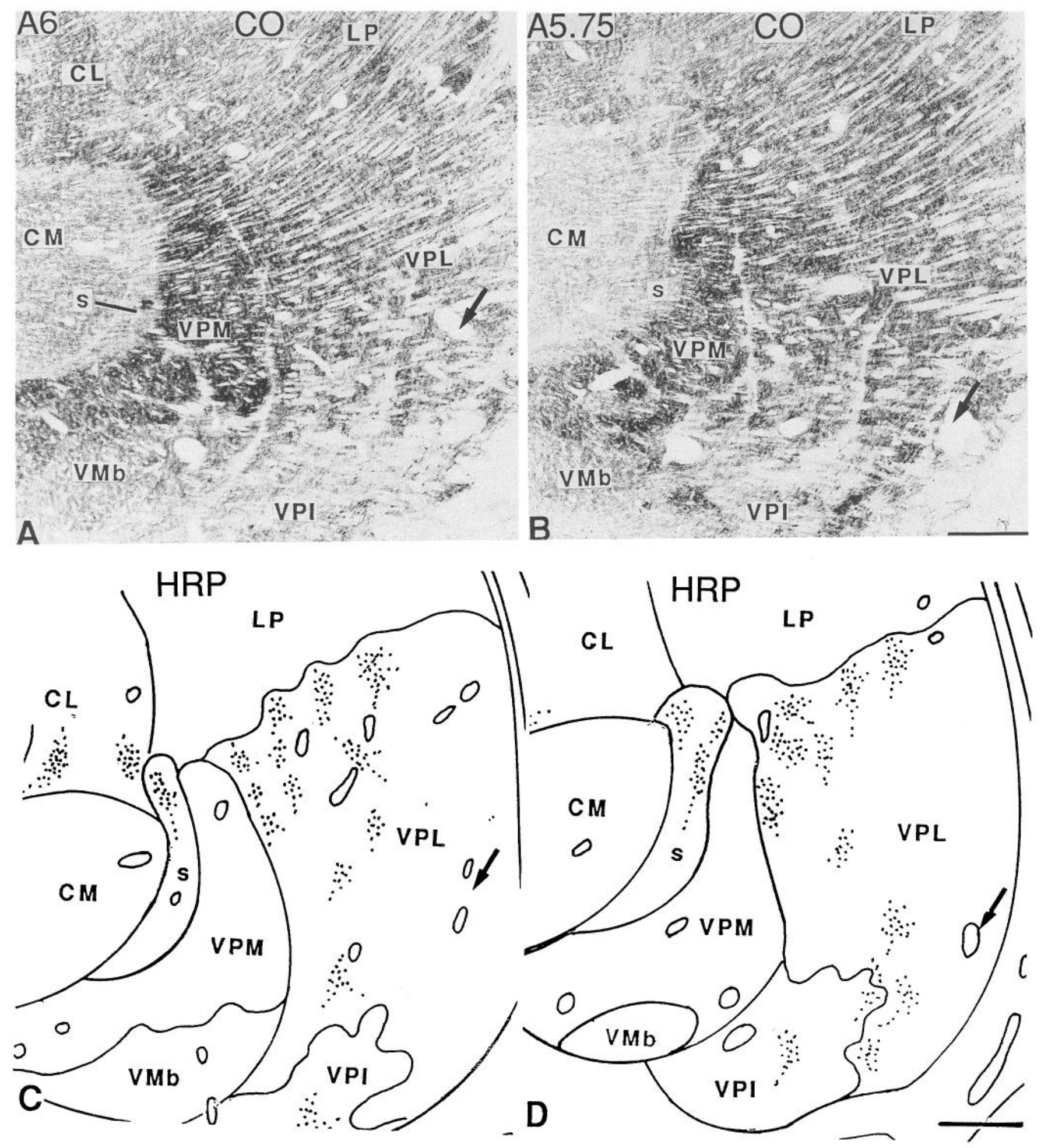

Figure 12. A series of frontal sections in anterior to posterior order $(A, B, E, F)$ at approximate stereotaxic levels indicated, stained for CO activity and matched to camera lucida drawings of closely adjacent sections $(C, D, G, H)$ on which are plotted the distributions of terminal ramifications of spinothalamic fibers, labeled by injections of HRP into the second cervical spinal segment. Arrows indicate same blood vessels. Note concentrations of spinothalamic terminations in CO-weak regions, including the posterior cap, which enlarges as CO-rich VPM rods start to disappear $(F, H$; see also Fig. 13). For abbreviatons, see Appendix. Scale bars, $1 \mathrm{~mm}$.

in the monkey thalamus has been described in a number of studies (Mehler et al., 1960; Bowsher, 1961; Mehler, 1969; Kent and Kerr, 1975; Boivie, 1979; Berkley, 1980; Burton and Craig, 1983; Mantyh, 1983; Apkarian and Hodge, 1989a-c; Gingold et al., 1991). All of these agree on the dissociated, clumped, or archipelago-like nature of the terminations and show the existence of a significant concentration in the general region here defined as the CO-weak cap of VPM. Unfortunately, few previous accounts have been accompanied by photomicrographs of the cytoarchitecture of the region, the location of the ter- 

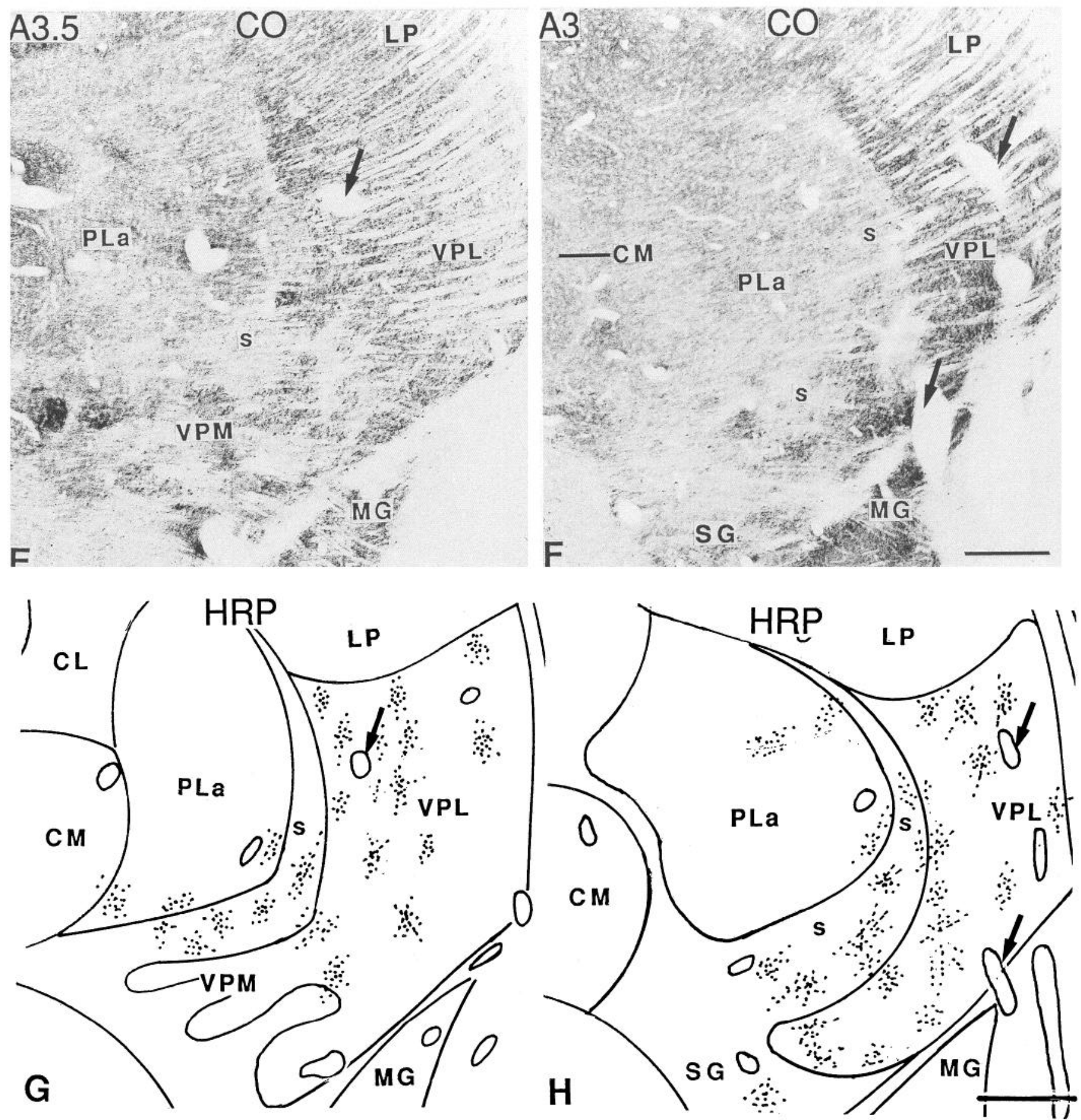

minations usually being shown on drawings derived from Olszewski's atlas (1952). In most cases, therefore, it is difficult to correlate the localization pattern with the underlying cyto- and chemoarchitecture revealed in the present study. Boivie (1979) shows a photomicrograph in which the small-celled cap is clearly revealed. This he designates the medial division of the posterior group of nuclei (Po) by analogy with appearances in the cat. Olszewski (1952) included a similar region in PLa. In the study of Gingold et al. (1991), a similar region is mainly incorporated into VPI and Po. Other studies, relying on atlas-based corre- lations, have located the posteromedial concentration in PLa, in a more caudal Po or SG or in VPL itself.

According to Ralston and Ralston (1992), the dorsal component of the spinothalamic tract terminates selectively in this caudal region. Past practice in this laboratory, following Olszewski, had been to designate the small-celled cap as part of PLa (Jones, 1985), but the present investigation and that of Rausell and Jones (1991a,b) has led to a reevaluation. The COstaining pattern is particularly useful in delineating the cap. Even when CO staining is weak, as in the small-celled matrix of VPM, 

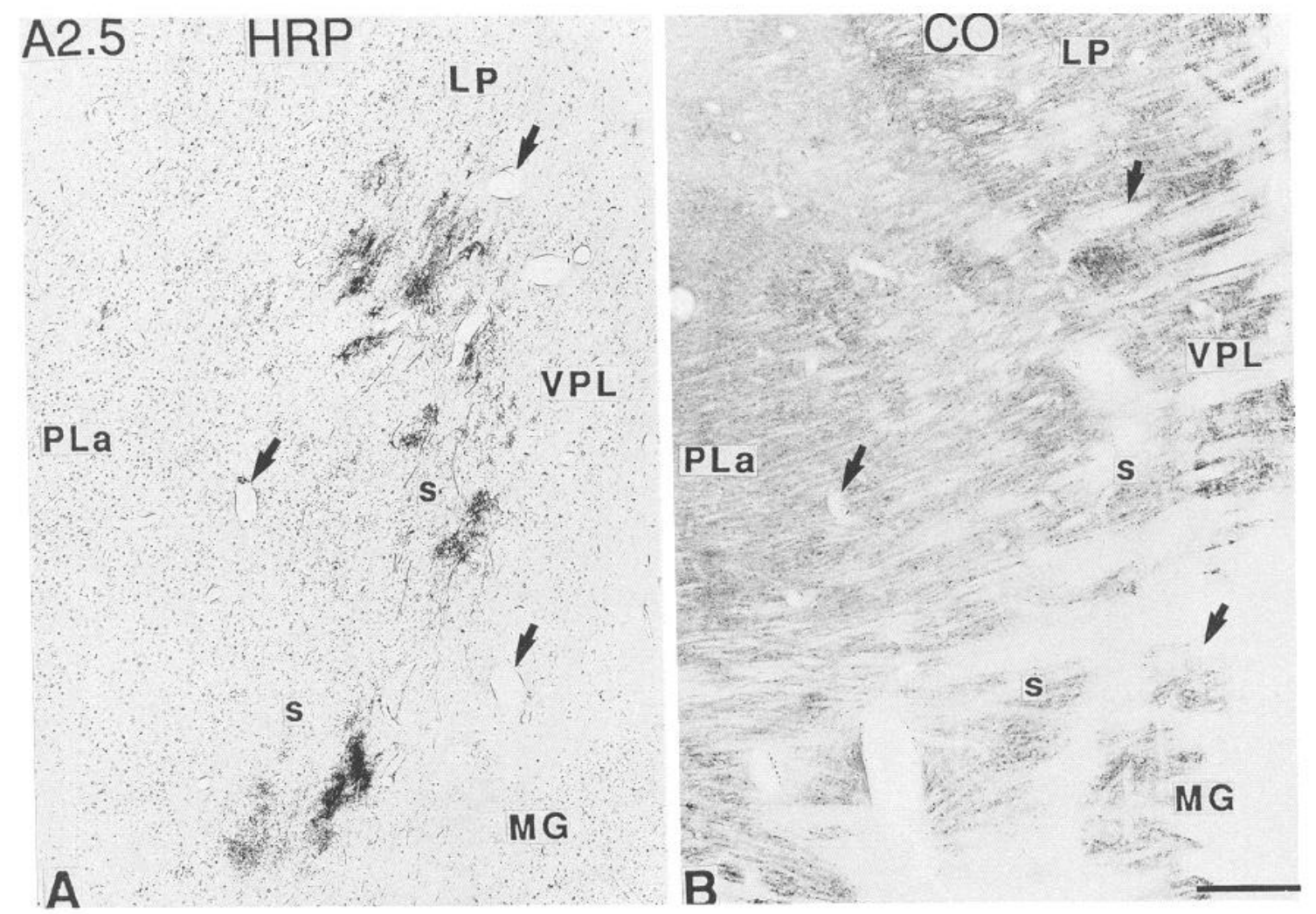

Figure 13. Sections from same brain as those shown in Figure 12 but at a more posterior level. $A$ shows spinothalamic terminations anterogradely labeled with HRP. The adjacent section $(B)$ stained for CO activity shows that most of these terminations are in the posterior cap (s). Arrows indicate same blood vessels. For abbreviatons, see Appendix. Scale bar, $1 \mathrm{~mm}$.

in the cap of VPM/VPL, and in PLa, there is still a degree of staining evident. Fiber laminae, by contrast, show no staining at all. On this basis, it is possible sometimes to detect a fine fiber lamina between PLa and the matrix of VPM anteriorly, although this disappears posteriorly. Distinguishing the cap from PLa posteriorly then depends mainly on the slightly denser CO staining and higher cell density in Nissl stains of PLa (see Fig. 4). There is a CO-negative fiber lamina separating VPM from VPL anteriorly, but at the posterior pole of VPM where the COdense rods of VPM start to disappear, this lamina breaks down and the CO-weak, small-celled zones of VPL and the expanding matrix of VPM merge to form the cap. For this reason, it seems appropriate, provisionally, to consider the cap a part of the ventral posterior nuclear complex.

An alternative viewpoint, previously expressed in reports describing studies on VPM (Rausell and Jones, 1991a,b), is to view the small-celled, CO-weak matrix of VPM, the cap, and CO-weak zones of VPL as part of a more widely distributed, $\mathrm{CO}$-weak region made up of smaller, calbindin-positive cells that is unconstrained by nuclear borders and extends virtually uninterruptedly from the posterior nucleus, through PLa, the CO-weak matrix of VPM, the CO-weak zones of VPL, and parts of CL into the VLp nucleus. The attraction of this interpretation is that this CO-weak, calbindin-rich region is virtually coextensive with the region of termination of the spinothalamic and caudal trigeminothalamic pathways. Possibly this entire region consists of layer I-projecting cells, but data are only available for the calbindin cells of VPM, VPL, and VLp. Although PLa is known to project to area 2 of SI and to area 5 (Burton and Jones, 1976; Jones et al., 1979; Pons and Kaas, 1985; Cusick and Gould, 1990), terminations of its axons have previously only been localized in layer III (Jones and Burton, 1976).

An even more radical view that will require confirmation would be to see the small calbindin-positive cells as a diffuse, layer I-projecting matrix of the entire thalamus upon which the classical nuclei, based upon cortical area-specific projections and terminations of major afferent pathways, are superimposed.

The two subnuclei (VPL and VPM) of the primate ventral posterior thalamic nucleus or ventrobasal complex, apart from intrinsic GABAergic neurons (Penny et al., 1983; Jones and Hendry, 1989; Benson et al., 1991; Hunt et al., 1991), appear to be composed essentially of two principal populations of relay neurons that selectively express calbindin or parvalbumin. The small number of neurons that coexpress parvalbumin and calbindin await further detailed evaluation, as does any potential breakdown of the calbindin cells into calbindin- and calretinin(Jacobowitz and Winsky, 1991) containing types. The two major populations now seem established as having laminar-specific projections to the somatosensory cortex - calbindin cells to layer I and parvalbumin cells to deeper layers. Deeper-layer projections of calbindin cells cannot be ruled out: although labeling of these cells after deep injections could be due to involvement of fibers passing through to layer $I$, it could also be due to projections to the middle layers as well. In the matrix of VPM and the posterior cap of VPL/VPM, the layer I-projecting cells are preferentially innervated by caudal trigeminothalamic and spinothalamic fibers. The spinothalamic system therefore appears to have preferential access to superficial layers of the somatosensory cortex from these regions. Calbindin cells in the remainder of VPL also project to layer $\mathrm{L}$, and those situated in 

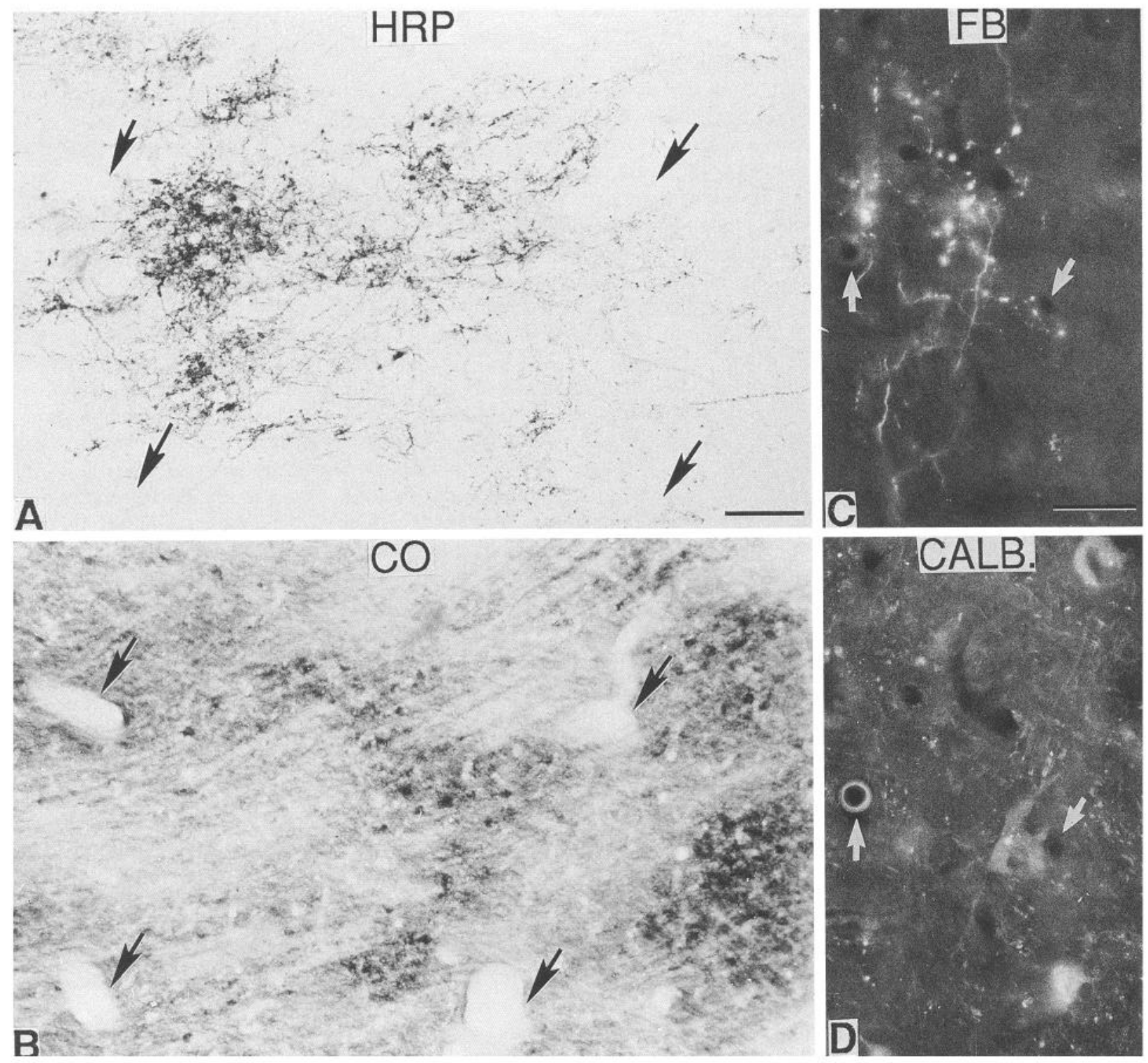

Figure 14. $A$ and $B$, The same parts of adjacent sections close to the posterior pole of VPM, showing HRP-labeled spinothalamic terminal fiber ramifications $(A)$ and $\mathrm{CO}$ activity $(B)$. Arrows indicate same blood vessels. Labeled fiber ramifications are concentrated in CO-weak regions. $C$ and $D$, Fluorescence micrographs from the same microscopic field of another brain, showing FB fluorescence in anterogradely labeled spinothalamic terminations $(C)$ in a region of calbindin-immunoreactive cells $(D)$. Fibers are not calbindin positive. Arrows indicate same blood vessels. Scale bars: $A$ and $B, 100 \mu \mathrm{m} ; C$ and $D, 25 \mu \mathrm{m}$.

CO-weak, parvalbumin cell-negative zones may also be primarily innervated by spinothalamic fibers, but this is not yet completely clear. Moreover, numerous layer I-projecting calbindin cells throughout VPL occupy parts of CO-rich regions containing numerous parvalbumin cells, innervated by lemniscal terminations and into which spinothalamic fiber terminations can intrude. While in some regions of VPL, therefore, layer I-projecting calbindin cells may receive a preponderance of spinothalamic inputs, in the majority they are likely to receive both spinothalamic and lemniscal terminals. A brief report of electron microscopic observations by Ralston (1991) indicates the presence of the two types of terminals on certain VPL neurons in the monkey.

The presence of both overlap and selectivity in the terminations of spinothalamic afferents on thalamic cells is not surprising in view of the reports of single-unit investigations in
VPL. Most parent neurons of the spinothalamic tract in monkeys are nociceptive but include wide-dynamic-range neurons that receive inputs from both nociceptors and low-threshold mechanoreceptors, and neurons that receive nociceptive-specific inputs (Willis et al., 1974; Price et al., 1978; Chung et al., 1979; Kenshalo et al., 1979; Surmeier et al., 1988). This is reflected in the stimulus-response characteristics of nociceptive thalamic neurons in monkeys (Perl and Whitlock, 1961; Pollin and Albe Fessard, 1979; Kenshalo et al., 1980; Casey and Morrow, 1983; Chung et al., 1986a,b). Neurons responding to noxious thermal or mechanical stimuli are greatly outnumbered by those responding only to innocuous stimuli. Among those responding to noxious stimuli, both wide-dynamic-range and highthreshold types are found. In the majority of cases, the nociceptive responses depend upon pathways in the lateral funiculus of the spinal cord (Chung et al., 1986b), suggesting specificity 
of innervation of the majority of these thalamic cells by spinothalamic afferents. However, responses of some nociceptive cells depend upon more dorsally situated spinal pathways (Chung et al., 1986b), and certain studies suggest that both dorsal column and lateral quadrant pathways in the spinal cord can converge on single thalamic neurons (Perl and Whitlock, 1961). Hence, a failure to detect a complete dissociation of spinothalamic terminations from CO-rich, lemniscal termination zones in the core of VPL is probably not unexpected. No studies have specifically reported on the responses of neurons in the VPM matrix or the posterior VPL/VPM cap, although Bushnell and Duncan (1987) have indicated the presence of cells in VPM with responses resembling those of superficial, caudal trigeminal neurons. A search for nociceptive responses in the matrix/map region might be revealing, especially since the cap is continuous with $\mathrm{Po}$ and is located in a region that suggests a correspondence with regions of the human thalamus in which electrical stimulation elicits thermal or painful sensations (Hassler, 1959, 1970; Halliday and Logue, 1972; Tasker et al., 1976, 1983; Lenz et al., 1987, 1988a,b), and in which lesions may alleviate painful states (Hassler and Reichert, 1959; Hassler, 1960). The cap also shows enhanced calbindin immunoreactivity in monkcys subjected to chronic dorsal rhizotomies leading to transneuronal degeneration of the parvalbumin-containing lemniscal system (Rausell et al., 1992). This has led to the suggestion that levels of the protein may be activity dependent, increasing in situations associated with an imbalance in the activity of the spinothalamic and lemniscal systems.

The projection of calbindin relay cells in VPL and VPM to the most superficial layer or layers of the somatosensory cortex suggests that input from these cells can potentially gain access to large populations of cortical neurons situated in all layers, since the pyramidal cells of layers II-VI have substantial dendritic ramifications in layer I of SI (Ramón y Cajal, 1899). A population of layer I-III GABAergic neurons with strong vertical axonal connections to subjacent layers also has dendritic access to layer I (DeFelipe et al., 1990). Hence, inputs to layer I are in a potentially powerful position to modulate levels of activity in all cortical layers.

Nociceptive-specific and wide-dynamic-range neurons have been described in both the face and body representations in the somatosensory cortex of anesthetized and conscious monkeys (Kenshalo and Isensee, 1983; Kenshalo et al., 1988; Chudler et al., 1990). The majority of nociceptive responsive cells were located in layer IV in these studies, but responding cells were also present in layers II-III and V. In general, the nociceptive responsive cells tended to be mingled with low-threshold cells. These responses are likely to be mediated by direct inputs to layer IV instead of to layer I. Thus, if the layer I-projecting calbindin system is associated with the central pain pathways, its role may be part of an arousal system rather than one that has the prime responsibility for the relay of information pertaining to the quality, intensity, and localization of a noxious stimulus. The immunocytochcmical characteristics of VPL nociceptive cells projecting to layer IV will be examined in future investigations.

Parvalbumin immunoreactivity defines the relay cells and their axons at all levels of the dorsal column and trigeminallemniscal system in primates, being found in fibers of the dorsal columns, in cells of the dorsal column and principal trigeminal nuclei, in fibers of the medial lemniscus, and in the thalamic cells among which these fibers terminate (Rausell and Jones, 1991a,b; Rausell et al., 1992). Calbindin immunoreactivity, however, cannot be regarded as a similar generic marker for the spinothalamic system. Although expressed in many cells in the marginal zone and substantia gelatinosa of the dorsal horn of primates (Fournet et al., 1986; Rausell et al., 1992) and other species (Celio, 1990), the cell distribution is not coextensive with the distribution of the cells of origin of the spinothalamic tract in primates (Albe-Fessard et al., 1974; Trevino and Carstens, 1975; Willis et al., 1979; Hayes and Rustioni; 1980; Apkarian and Hodge, 1989a,b). Moreover, it was not possible to identify calbindin-immunoreactive, FB-labeled spinothalamic fibers entering the thalamus in the present study, and a search for calbindin-immunoreactive dorsal horn cells retrogradely labeled with $\mathrm{FB}$ in the same animals was negative (E. Rausell and E. G. Jones, unpublished observations).

The functions of the calcium-binding proteins in neurons remain largely unknown. Earlier correlations with the buffering of calcium associated with the fast spiking behavior of certain neuronal populations and a specific localization in GABAergic neurons (Celio, 1986; Kawaguchi et al., 1987; Kosaka et al., 1987) did not reveal the full picture: There are substantial regional and species differences in the association with GABA cells and in the differential expression of calbindin or parvalbumin. For example, in the cerebral cortex of all species examined, parvalbumin immunoreactivity is found in one population of GABA cells while calbindin immunoreactivity is found in a second population of GABA cells and in certain pyramidal cells (Hendry et al., 1989; Demeulemeester et al., 1991; DeFelipe and Jones, 1992). In the thalamus of all species, parvalbumin colocalizes with GABA immunoreactivity in the reticular nucleus (Jones and Hendry, 1989). In the dorsal thalamus, however, parvalbumin is specifically associated with intrinsic GABA cells in cats (Stichel et al., 1988), while neither calcium-binding protein is found to a significant extent in intrinsic GABA neurons of the monkey (Jones and Hendry, 1989). At the present time, the calcium-binding proteins are useful markers for cell identity, for nuclear delineations and for patterns of connectivity, but determining their different functions, if any, remains a challenge.

\section{Appendix}

List of anatomical abbreviations

CL Central lateral nucleus

$\mathrm{CM} \quad$ Centre médian nucleus

LP Lateral posterior nucleus

MG Medial geniculate nucleus

PLa Anterior pulvinar nucleus

Po Posterior nucleus

$\mathrm{R} \quad$ Reticular nucleus

SG Suprageniculate nucleus

s Small-celled, CO-weak region

VLa Ventral lateral anterior nucleus

VLp Ventral lateral posterior nucleus

$\mathrm{VMb} \quad$ Basal ventral medial nucleus

VPI Ventral posterior inferior nucleus

VPL Ventral posterior lateral nucleus

VPM Ventral posterior medial nucleus

\section{References}

Albe-Fessard D, Levante A, Lamour Y (1974) Origin of spinothalamic tract in monkeys. Brain Res 65:503-509.

Apkarian AV, Hodge CJ (1989a) The primate spinothalamic pathways: I. A quantitative study of the cells of origin of the spinothalamic pathway. J Comp Neurol 288:447-473. 
Apkarian AV, Hodge CJ (1989b) The primate spinothalamic pathways: II. The cells of origin of the dorsolateral and ventral spinothalamic pathways. J Comp Neurol 288:474-492.

Apkarian AV, Hodge CJ $(1989 \mathrm{c})$ The primate spinothalamic pathways: III. Thalamic terminations of the dorsolateral and ventral spinothalamic pathways. J Comp Neurol 288:493-511.

Applebaum AE, Leonard RB, Kenshalo DR, Martin RF, Willis WD (1979) Nuclei in which functionally identified spinothalamic tract neurons terminate. J Comp Neurol 188:575-586.

Asanuma C, Thach WT, Jones EG (1983) Distribution of cerebellar terminations and their relation to other afferent termination in the ventral lateral thalamic region of the monkey. Brain Res Rev 5:237265.

Avendaño C, Stepniewska I, Rausell E, Reinoso-Suarez F (1990) Segregation and heterogeneity of thalamic cell populations projecting to superficial layers of posterior parietal cortex: a retrograde tracer study in cat and monkey. Neuroscience 39:547-560.

Benson DL, Isackson PJ, Hendry SHC, Jones EG (1991) Differential gene expression for glutamic acid decarboxylase and type II calciumcalmodulin-dependent protein kinase in basal ganglia, thalamus and hypothalamus of the monkey. J Neurosci 11:1540-1564.

Berkley K (1980) Spatial relationships between the termination of somatic sensory and motor pathways in the rostral brainstem of cats and monkeys: I. Ascending somatic sensory inputs to lateral diencephalon. J Comp Neurol 193:283-317.

Birnbacher D, Albus K (1987) Divergence of single axons in afferent projections to the cat's visual cortex. J Comp Neurol 261:543-561.

Boivie J (1978) Anatomical observations on the dorsal column nuclei, their thalamic projection and the cytoarchitecture of some somatosensory thalamic nuclei in the monkey. J Comp Neurol 178:17-48.

Boivie J (1979) An anatomical reinvestigation of the termination of the spinothalamic tract in the monkey. J Comp Neurol 186:343-370.

Bowsher D (1961) The termination of secondary somatosensory neurons within the thalamus of Macaca mulatta: an experimental degeneration study. J Comp Neurol 117:213-227.

Burton H, Craig AD (1983) Spinothalamic projections in cat, raccoon and monkey: a study based on anterograde transport of horseradish peroxidase. In: Somatosensory integration in the thalamus (Macchi G, Rustioni A, Spreafico A, eds), pp 1-25. Amsterdam: Elsevier.

Burton H, Jones EG (1976) The postcrior thalamic region and its cortical projection in new world and old world monkeys. J Comp Neurol 168:249-302.

Bushnell MC, Duncan GH (1987) Mechanical response properties of ventroposterior medial thalamic neurons in the alert monkey. Exp Brain Res 67:603-614.

Casey KL, Morrow TJ (1983) Ventral posterior thalamic neurons differentially responsive to noxious stimulation of the awake monkey. Science 221:675-677.

Celio MR (1986) Parvalbumin in most $\gamma$-aminobutyric acid-containing neurons of the rat cerebral cortex. Science 231:995-997.

Celio MR (1990) Calbindin D-28k and parvalbumin in the rat nervous system. Neuroscience 35:375-475.

Chudler EH, Anton F, Dubner R, Kenshalo DR Jr (1990) Responses of nociceptive SI neurons in monkeys and pain sensation in humans elicited by noxious thermal stimulation: effect of interstimulus interval. J Neurophysiol 63:559-569.

Chung JM, Kenshalo DR Jr, Gerhart KD, Willis WD (1979) Excitation of primate spinothalamic neurons by cutaneous C-fiber volleys. J Neurophysiol 42:1354-1369.

Chung JM, Surmeier DJ, Lee KH, Sorkin LS, Honda CN, Tsong Y, Willis WD (1986a) Classification of primate spinothalamic and somatosensory thalamic neurons based on cluster analysis. I Neurophysiol 56:308-327.

Chung JM, Lee KH, Surmeier DJ, Sorkin LS, Kim J, Willis WD (1986b) Response characteristics of neurons in the ventral posterior lateral nucleus of the monkey thalamus. J Neurophysiol 56:370-390.

Cleland BG, Dubin MW, Levick WR (1971) Sustained and transient neurones in the cat's retina and lateral geniculate nucleus. J Physiol (Lond) 217:473-496.

Cusick CG, Gould HJ III (1990) Connections between area 3b of the somatosensory cortex and subdivisions of the ventroposterior nuclear complex and the anterior pulvinar nucleus in squirrel monkeys. $\mathrm{J}$ Comp Neurol 292:83-102.

DeFelipe J, Hendry SHC, Hashikawa T, Molinari M, Jones EG (1990) A microcolumnar structure of monkey cerebral cortex revealed by immunocytochemical studies of double bouquet cell axons. Ncuroscience 37:655-673.

DeFelipe J, Jones EG (1992) High-resolution light and electron microscopic immunocytochemistry of colocalized GABA and calbindin D-28k in somata and double bouquet cell axons of monkey somatosensory cortex. Eur J Neurosci 4:46-60.

DeHoff R'T, Rhines FN (1961) Determination of the number of particles per unit volume from measurements made on random plane sections: the general cylinder and the ellipsoid. Trans Amer Inst Metall 221:975-982.

Demeulemeester H, Arckens L, Vandesande F, Orban GA, Heizmann $\mathrm{CW}$, Pochet $\mathrm{R}$ (1991) Calcium binding proteins as molecular markers for cat geniculate neurons. Exp Brain Res 83:513-520.

Fournet N, Garcia-Segura LM, Norman AW, Orci L (1986) Selective localization of calcium-binding protein in human brainstem, cerebellum and spinal cord. Brain Res 399:310-316.

Friedlander MJ, Lin GS, Stanford LR, Sherman SM (1981) Morphology of functionally identified neurons in the lateral geniculate nucleus of the cat. J Neurophysiol 46:80-129.

Gingold SI, Greenspan JD, Apkarian A (1991) Anatomic evidence of nociceptive inputs to primary somatosensory cortex: relationship between spinothalamic terminals and thalamocortical cells in squirrel monkeys. J Comp Neurol 308:467-490.

Gordon GG, Manson JR (1967) Cutaneous receptive fields of single nerve cells in the thalamus of the cat. Nature 215:597-599.

Halliday $\Lambda M$, Logue V (1972) Painful sensations evoked by electrical stimulation in the thalamus. In: Neurophysiology studied in man (Somjen GG, ed), pp 221-230. Amsterdam: Excerpta Medica.

Hashikawa T, Rausell E, Molinari M, Jones EG (1991) Parvalbuminand calbindin-containing neurons in the monkey medial geniculate complex: differential distribution and cortical layer specific projections. Brain Res 544:335-341.

Hassler R (1959) Anatomy of the thalamus. In: Introduction to stereotaxis with an atlas of the human brain (Schaltenbrand G, Bailey $P$, eds), pp 230-290. Stuttgart: Thieme.

Hassler R (1960) Die zentralen System des Schmerzes. Acta Neurochir $8: 353-423$.

Hassler R (1970) Dichotomy of facial pain conduction in the diencephalon. In: Trigeminal neuralgia (Hassler $\mathrm{R}$, Walker AE, eds), pp 123-138. Stuttgart: Thieme

Hassler R, Riechert T (1959) Klinische und anatomische Befunde bei stereotaktischen Schmerzoperationen im Thalamus. Arch Psychiat Nervenkr 200:93-122.

Hayes NL, Rustioni A (1980) Spinothalamic and spinomedullary neurons in macaques: a single and double retrograde tracer study. Neuroscience 5:861-874.

Hendry SHC, Jones EG, Hockfield S, McKay RDG (1988) FBNeuronal populations stained with the monoclonal antibody Cat-301 in the mammalian cerebral cortex and thalamus. J Neurosci 8:518-542.

Hendry SHC, Jones EG, Emson PC, Lawson DEM, Heizmann CW, Streit $\mathbf{P}$ (1989) Two classes of cortical GABA neurons defined by differential calcium binding protein immunoreactivities. Exp Brain Res 76:467-472.

Herkenham M (1986) New perspectives on the organization of nonspecific thalamocortical projections. In: Cerebral cortex, Vol 5, Sensory-motor areas and aspects of cortical connectivity (Jones EG, Peters A, eds), pp 403-446. New York: Plenum.

Hockfield S, Sur M (1990) Monoclonal antibody: Cat 301 identifies Y-cells in the dorsal lateral geniculate nucleus of the cat. J Comp Neurol 300:320-330.

Hunt CA, Pang DZ, Jones EG (1991) fbDistribution and density of GABA cells in intralaminar and adjacent nuclei of monkey thalamus. Neuroscience 43:185-196.

Jacobowitz DM, Winsky L (1991) Immunocytochemical localization of calretinin in the forebrain of the rat. J Comp Neurol 304:198-218.

Jones EG (1985) The thalamus. New York: Plenum.

Jones EG, Burton H (1976) Areal differences in the laminar distribution of thalamic afferents in cortical fields of the insular, parictal and temporal regions of primates. J Comp Neurol 168:197-248.

Jones EG, Friedman DP (1982) Projection pattern of functional components of thalamic ventrobasal complex on monkey somatosensory cortex. J Neurophysiol 48:521-544.

Jones EG, Hendry SHC (1989) Differential calcium binding protein immunoreactivity distinguishes classes of relay neurons in monkey thalamic nuclei. Eur J Neurosci 1:222-246. 
Jones EG, Wise SP, Coulter JD (1979) Differential thalamic relationships of sensory-motor and parietal cortical fields in monkeys. J Comp Neurol 183:833-882.

Jones EG, Friedman DP, Hendry SHC (1982) Thalamic basis of place and modality-specific columns in monkey somatosensory cortex: a correlative anatomical and physiological study. J Neurophysiol 48: 545-568.

Jones EG, Hendry SHC, Brandon C (1986a) Cytochrome oxidase staining reveals functional organization of monkey somatosensory thalamus. Exp Brain Res 62:438-442.

Jones EG, Schwark HD, Callahan PJ (1986b) Extent of the ipsilateral representation in the ventral posterior medial nucleus of the monkey thalamus. Exp Brain Res 63:310-320.

Kaas JH, Nelson RJ, Sur M, Dykes RW, Merzenich MM (1984) The somatotopic organization of the ventroposterior thalamus of the squirrel monkey Saimiri sciureus. J Comp Neurol 226:111-140.

Kaske A, Dick A, Creutzfeldt OD (1991) The local domain for divergence of subcortical afferents to the striate and extrastriate visual cortex in the common marmoset (Callithrix jacchus): a multiple labelling study. Exp Brain Res 84:254-265.

Kawaguchi Y, Katsumaru H, Kosaka T, Heizmann CW, Hama K (1987) Fast spiking cells in rat hippocampus (CA1 region) contain the calcium-binding protein parvalbumin. Brain Res 416:369-374.

Kenshalo DR, Isensee O (1983) Responses of primate SI cortical neurons to noxious stimuli. J Neurophysiol 50:1479-1496.

Kenshalo DR, Leonard RB, Chung JM, Willis WD (1979) Responses of primate spinothalamic neurons to graded and to repeated noxious heat stimuli. J Neurophysiol 42:1370-1389.

Kenshalo DR Jr, Giesler GJ Jr, I eonard RB, Willis WD (1980) Responses of neurons in primate ventral posterior lateral nucleus to noxious stimuli. J Neurophysiol 43:1594-1614.

Kenshalo DR, Chudler EH, Anton F, Dubner R (1988) SI nociceptive neurons participate in the encoding process by which monkeys perceive the intensity of noxious thermal stimulation. Brain Res 454: 378-382.

Kerr FWL (1975) The ventral spinothalamic tract and other ascending systems of the ventral funiculus of the spinal cord. J Comp Neurol 159:335-356.

Kerr FWL, Lippman HH (1974) The primate spinothalamic tract as demonstrated by anterolateral cordotomy and commissural myelotomy. In: Advances in neurology (Bonica JJ, ed), pp 147-156. New York: Raven.

Kosaka T, Katsumaru H, Hama K, Wu J-Y, Heizmann CW (1987) GABAergic neurons containing the $\mathrm{Ca}^{2+}$-binding protein parvalbumin in the rat hippocampus and dentate gyrus. Brain Res 419:119130.

LeGros Clark WE (1932) The structure and connections of the thalamus. Brain 55:406-470.

Lenz FA, Tasker RR, Dostrovsky JO, Kwan HC, Gorecki J, Hirayama T, Murphy JT (1987) Abnormal single-unit activity recorded in the somatosensory thalamus of a quadriplegic patient with central pain. Pain 31:225-236.

Lenz FA, Dostrovsky JO, Tasker RR, Yamashiro K, Kwan HC, Murphy JT (1988a) Single-unit analysis of the human ventral thalamic nuclear group: somatosensory responses. J Neurophysiol 59:299-316.

Lenz FA, Tasker RR, Dostrovsky JO, Kwan HC, Gorecki J, Hirayama T, Murphy JT (1988b) Abnormal single-unit activity and responses to stimulation in the presumed ventrocaudal nucleus of patients with central pain. In: Proceedings of the Vth World Congress on Pain (Gebhardt GF, Bond MR, Dubner R, cds), pp 157-164. Amsterdam: Elsevier.

Macchi G (1988) Thalamic entities and the problem of thalamocortical integration. In: Cellular thalamic mechanisms (Bentivoglio M, Spreafico R, eds), pp 569-588. Amsterdam: Elsevier.

Macchi G, Bentivoglio M, Molinari M, Minciacchi D (1984) The thalamocaudate versus thalamo-cortical projections as studied in the cat with fluorescent retrograde double labeling. Exp Brain Res 54: 225-239.

Mantyh PW (1983) The spinothalamic tract in the primate: a reexamination using wheatgerm agglutinin conjugated to horseradish peroxidase. Neuroscience 9:847-862.

Mehler WR (1969) Some neurological species differences; a posteriori. Ann NY Acad Sci 167:424-468.

Mehler WR, Feferman ME, Nauta WJ (1960) Ascending axon degeneration following anterolateral cordotomy: experimental study in the monkey. Brain 83:718-750.
Mesulam M-M (1978) Tetramethylbenzidine for horseradish peroxidase neurohistochemistry: a non-carcinogenic blue reaction product with superior sensitivity for visualizing neural afferent and efferents. J Histochem Cytochem 26:106-117.

Minciacchi D, Bentivoglio M, Molinari M, Kultas-Ilinsky K, Ilinsky IA, Macchi G (1986) Multiple cortical targets of one thalamic nucleus: the projections of the ventral medial nucleus in the cat studied with retrograde tracers. J Comp Neurol 252:106-129.

Montero VM, Zempel J (1986) The proportion and size of GABAimmunoreactive neurons in the magnocellular and parvocellular layers of the lateral geniculate nucleus of the rhesus monkey. Exp Brain Res 62:215-223.

Morel A, Imig TJ (1987) Thalamic projections to fields A, AI, P and VP in the cat auditory cortex. J Comp Neurol 265:119-144.

Mountcastle VB, Henneman N (1952) The representation of tactile sensibility in the thalamus of the monkey. J Comp Neurol 97:409440 .

Mountcastle VB, Poggio GF (1963) The functional properties of ventrobasal thalamic neurons studied in unanesthetized monkeys. J Neurophysiol 26:775-806.

Olszewski J (1952) The thalamus of the Macaca mulatta. An atlas for use with the stereotaxic instrument. New York: Karger.

Penny GR, Itoh K, Diamond IT (1982) Cells of different sizes in the ventral nuclei project to different layers of the somatic cortex in the cat. Brain Res 242:55-66.

Penny GR, Fitzpatrick D, Schmechel DE, Diamond IT (1983) Glutamic acid decarboxylase-immunoreactive neurons and horseradish peroxidase-labeled projection neurons in the ventral posterior nucleus of the cat and Galago senegalensis. J Neurosci 3:1868-1887.

Perl ER, Whitlock DG (1961) Somatic stimuli exciting spinothalamic projections to thalamic neurons in cat and monkey. Exp Neurol 3: 256-296.

Pollin B, Albe-Fessard D (1979) Organization of somatic thalamus in monkeys with and without section of dorsal spinal tracts. Brain Res 173:431-449.

Pons TP, Kaas JH (1985) Connections of area 2 of somatosensory cortex with the anterior pulvinar and subdivisions of the ventroposterior complex in macaque monkeys. J Comp Neurol 240:16-36.

Price DD, Hayes RL, Ruda M, Dubner R (1978) Spatial and temporal transformations of input to spinothalamic tract neurons and their relation to somatic sensations. J Neurophysiol 41:933-947.

Ralston HJ III (1969) The synaptic organization of lemniscal projections to the ventrobasal thalamus in the cat. Brain Res 14:99-116.

Ralston HJ III (1991) Local circuitry of the somatosensory thalamus in the processing of sensory information. In: Progress in brain research, Vol 87, Role of the forebrain in sensation and behavior $(\mathrm{Hol}$ stege G, ed), pp 13-28. Amsterdam: Elsevier.

Ralston HJ, Ralston DD (1992) The primate dorsal spinothalamic tract: evidence for a specific termination in the posterior nuclei $(\mathrm{Po} /$ SG) of the thalamus. Pain 48:107-118.

Ramón y Cajal S (1899) Estudios sobre la corteza cerebral humana. II: Estructura de la corteza motriz del hombre y mamíferos superiores. Rev Trim Micrográf 4:117-200.

Rausell E, Avendaño C (1985) Thalamocortical neurons projecting to superficial and to deep layers in parietal, frontal and prefrontal regions in the cat. Brain Res 347:159-165.

Rausell E, Jones EG (1991a) Histochemical and immunocytochemical compartments of the thalamic VPM nucleus in monkeys and their relationship to the representational map. J Neurosci 11:210-225.

Rausell E, Jones EG (1991b) Chemically distinct compartments of the thalamic VPM nucleus in monkeys relay principal and spinal trigeminal pathways to different layers of the somatosensory cortex. J Neurosci 11:226-237.

Rausell E, Bae C-S, Cusick CG, Jones EG (1991) Chemical compartments in VPL nucleus of monkey thalamus. Soc Neurosci Abstr 17: 625

Rausell E, Cusick CG, Taub E, Jones EG (1992) Chronic deafferentation in monkeys differentially affects nociceptive and nonnociceptive pathways distinguished by specific calcium-binding proteins and down-regulates $\gamma$-aminobutyric acid type A receptors at thalamic levels. Proc Natl Acad Sci USA 89:2571-2575.

Rogers JH (1987) Calretinin: a gene for a novel calcium-binding protein expressed principally in neurons. J Cell Biol 105:1343-1353.

Stanford LR, Friedlander MJ, Sherman SM (1983) Morphological and physiological properties of geniculate W-cells in the cat. J Neurophysiol 50:582-608. 
Stichel CC, Singer W, Heizmann CW (1988) Light and electron microscopic immunocytochemical localization of parvalbumin in the dorsal lateral geniculate nucleus of the cat: evidence of co-existence with GABA. J Comp Neurol 268:29-37.

Surmeier DJ, Honda CN, Willis WD (1988) Natural groupings of primate spinothalamic neurons based on cutaneous stimulation. Physiological and anatomical features. J Neurophysiol 59:833-860.

Tasker RR, Organ LW, Rowe IH, Hawrylyshyn P (1976) Human spinothalamic tract-stimulation mapping in the spinal cord and brainstem. Adv Pain Res Ther 1:251-257.

Tasker RR, Tsuda T, Hawrylyshyn P (1983) Clinical neurophysiological investigation of deafferentation pain. Adv Pain Res Ther 5 713-738.

Tracey DJ, Asanuma C, Jones EG, Porter R (1980) Thalamic relay to motor cortex: afferent pathways from brainstem. cerebellum and spinal cord in monkeys $J$ Neurophysiol 44:532-554.

Trevino DL, Carstens E (1975) Confirmation of the location of spinothalamic neurons in the cat and monkey by retrograde transport of horseradish peroxidase. Brain Res 98:177-182.
Walker AE (1938) The primate thalamus. Chicago: University of Chicago.

Wiener SI, Johnson JI, Ostapoff EM (1987) Demarcations of the mechanosensory projection zones in the raccoon thalamus, shown by cytochrome oxidase, acetylcholinesterase, and Nissl stains. J Comp Neurol 258:509-526.

Willis WD, Trevino DL, Coulter JD, Maunz RA (1974) Responses of primate spinothalamic tract neurons to natural stimulation of hindlimb. J Neurophysiol 37:358-372.

Willis WD, Kenshalo DR Jr, Leonard RB (1979) The cells of origin of the primate spinothalamic tract. J Comp Neurol 188:543-574.

Wong-Riley M (1979) Changes in the visual system of monocularly sutured or enucleated cats demonstrable with cytochrome oxidase histochemistry. Brain Res 171:11-28.

Yen C-T, Conley M, Jones EG (1985) Morphologic and functional types of neurons in cat ventral posterior thalamic nucleus. J Neurosci 5:1316-1338. 